\title{
CHAPTER 2.5.
}

\section{MATHEMATICAL MODELS FOR USING STERILE INSECTS}

\author{
H. J. BARCLAY
}

Victoria, B.C., Canada

Email:hbarclay@shaw.ca

\section{TABLE OF CONTENTS}

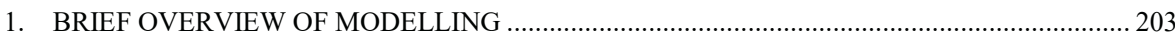

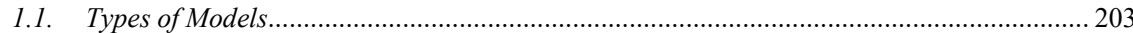

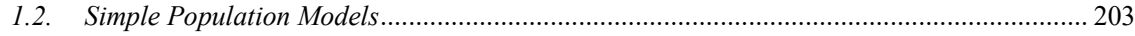

1.3. Host-Parasitoid and Predator-Prey Models................................................................... 204

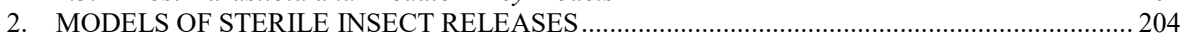

2.1. Three Kinds of Control Programmes Using Sterility ...................................................... 204

2.2. Initial Contribution of Knipling to Modelling the SIT ........................................................ 205

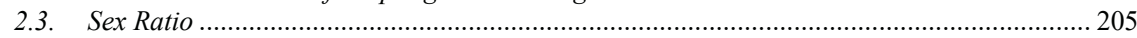

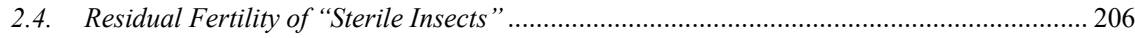

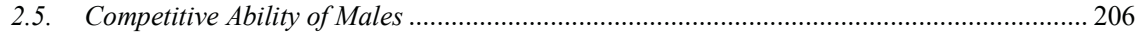

2.6. Interactions of Residual Fertility and Competitiveness .................................................... 208

2.6.1. Residually Fertile Insects Are Fully Competitive .............................................. 208

2.6.2. Residually Fertile Insects Have Reduced Competitiveness ................................... 208

2.7. Mating Patterns............................................................................................................. 208

2.7.1. Interaction of Polygamy with Competitive Ability ............................................ 209

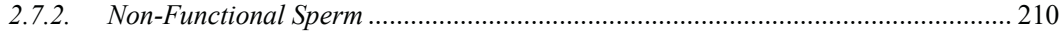

2.7.3. Dominant Lethal Mutations with Fully Competitive Sperm .................................. 210

2.7.4. Dominant Lethal Mutations with Reduced Sperm Function ................................ 211

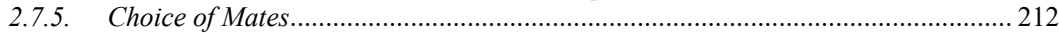

2.8. Interaction of Polygamy with Residual Fertility .............................................................. 212

2.9. Stochastic Models..................................................................................................... 213

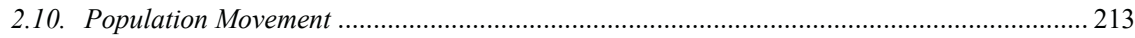

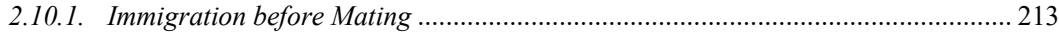

2.10.2. Immigration after Mating ........................................................................... 214

2.10.3. Combinations of Residual Fertility, Reduced Competitiveness, and Immigration .. 214

2.10.4. Large-Scale Population Movement …........................................................... 215

2.10.5. Effects of Movement of Individuals on Efficiency of the SIT .................................. 216

Pages 201-244

V. A. Dyck, J. Hendrichs and A. S. Robinson (eds.), Sterile Insect Technique. Principles and Practice in Area-Wide Integrated Pest Management. Second Edition.

(C) 2021 IAEA. CRC Press, Boca Raton, Florida, USA. 
2.10.6. Redistribution of Released Sterile Insects ...........................................................216

2.10.7. A Model for Eldana saccharina with Diffusion and Partial Sterility ........................217

2.11. Density-Dependence in Population Regulation ............................................................218

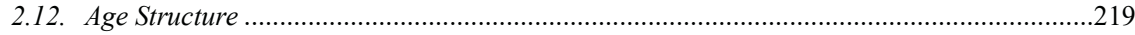

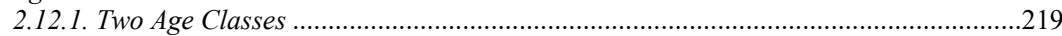

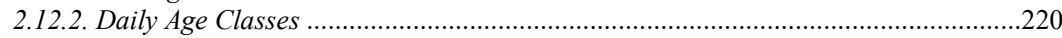

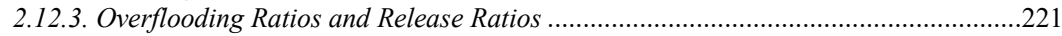

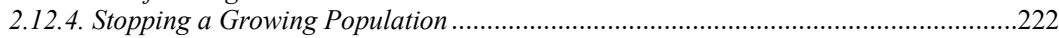

2.13. Population Aggregation ................................................................................................222

2.14. Predation, Parasitism, and Competition ....................................................................222

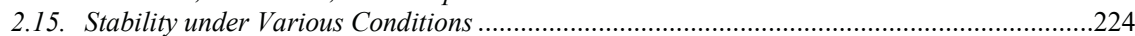

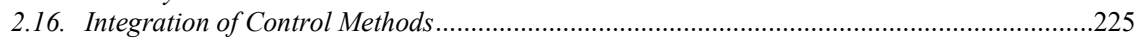

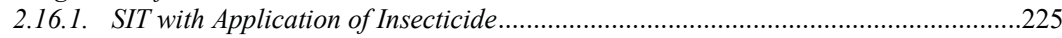

2.16.2. SIT with Pheromone Traps for Male Annihilation ...............................................226

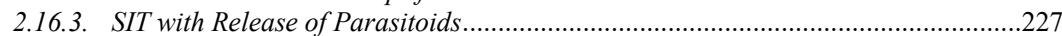

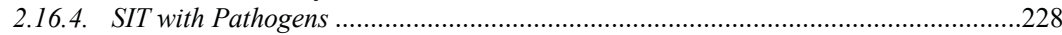

2.16.5. SIT with Sanitary Measures and Oviposition Traps .............................................229

2.16.6. Synergism between the SIT and a Natural Allee Effect ..........................................229

2.17. Optimizing Initial Knockdown for the SIT ....................................................................229

2.18. Exclusion of Pests from an Area: Barrier Width .............................................................230

2.18.1. Description of the Diffusion Model ......................................................................221

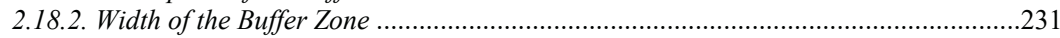

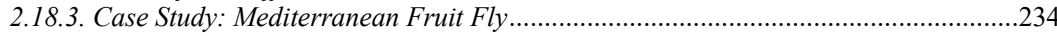

2.19. Optimization of Programme Releasing Sterile Insects ....................................................235

2.20. Development of Resistance ...............................................................................................236

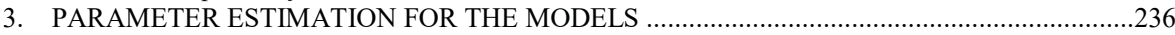

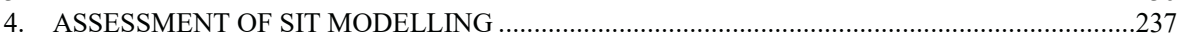

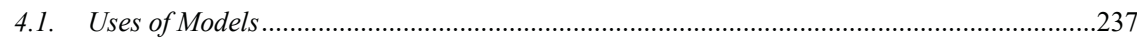

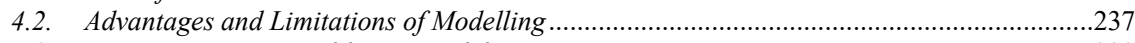

4.3. Transient versus Equilibrium Models .........................................................................238

4.4. Future Directions and Information Needs .....................................................................238

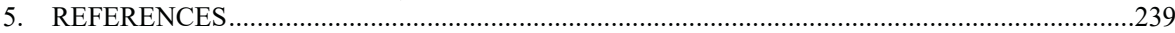

\section{SUMMARY}

This chapter begins with a consideration of simple population models. The sterility formulation proposed by Knipling is then included in the population models, and these are elaborated in systematic fashion to include the major biological factors that will affect the success of a sterile insect technique (SIT) control programme. These factors include residual fertility, differential competitive ability of wild and sterilized males, mating patterns, immigration, and various combinations of these features. Also examined are density-dependence, age structure, population aggregation, Allee effect, biotic interactions with other species, and then integration of the SIT with other control methods. It was found that combinations of factors are often synergistic: combinations of detrimental factors such as residual fertility and inferior competitive ability put severe limits on the probable success of the control programme, while combinations of control methods are much more likely to succeed than single control methods. This is because each control method needs only to account for a smaller proportion of the total mortality when combined with other methods than when acting alone. The barrier width is computed to protect an area from pests outside the controlled area using a diffusion equation, and a case study is presented using Mediterranean fruit flies. The redistribution of sterile males released from aircraft travelling along predetermined flight lines is also addressed using a diffusion equation. To minimize costs, a short method is presented to calculate the optimal frequency of releases, distance between flight lines, and number of sterile males released on each flight. This short method is capable of calculation using a hand calculator. Tentative results are also presented on optimizing the number of sprays and time intervals between sprays of a pest population in preparation for an SIT project. The question of whether to release only sterile males or both sexes is examined in the light of results for tephritids. Parameter estimation is mentioned, and also an assessment of the models presented herein. Finally, a brief section on future needs and trends for modelling the SIT is presented. 


\section{BRIEF OVERVIEW OF MODELLING}

\subsection{Types of Models}

Modelling is the abstraction of processes or states of being. Mathematical models involve equations, graphs or algorithms behind computer code. Virtually all models of the sterile insect technique (SIT) are population models, either analytic (just with equations) or computer models (often called numerical models and in which the equations are usually implicit, rather than being made explicit). Population models keep track of population numbers, and include various features that influence population size and trend, such as birth rate, mortality, age structure, immigration and emigration, competition, etc. Population growth can be either densityindependent, in which birth rate and mortality are independent of population size, or density-dependent, in which either or both of birth rate and mortality depend on population size, usually in such a way as to eventually stabilize the population around some long-term mean value.

Mathematical models of populations are typically posed as difference equations or as differential equations. Difference equations are discrete and use some meaningful time step, such as days, years, generations, etc. These are popular with entomologists, since many insects breed seasonally, such as most temperate forest insect pests (bark beetles, budworms, tent caterpillars, etc.). Differential equations are continuous, involving an infinitesimal time step, and rely on calculus to solve them. They are useful in species that breed continuously within some period of time, such as aphids, stored products pests and animal parasites. However, difference equations do not involve calculus, and generally are easier for the nonmathematician to understand. Another dichotomy is between deterministic models, which always yield the same model result, and stochastic models, which involve randomness. Most of the models of the SIT have so far been deterministic models, involving no random elements.

In this chapter, only models that predict some aspect of system behaviour (population dynamics) will be explored. Thus, purely statistical analysis of data, however valuable and relevant that might be to a release programme, will not be considered here. Likewise the derivation of regressions for use in models will not be considered here as SIT modelling. However, a section on model parameter estimation, in which such techniques are mentioned, is included.

\subsection{Simple Population Models}

For density-independent population growth, the simplest models are geometric growth for a species with non-overlapping generations, and its continuous counterpart, exponential growth (Box 1). A simple modification to these models, to include resource limitation, puts an upper limit on growth. Many formulations exist for limiting geometric growth; a few were provided by Hassell (1978). This small complication makes some formulations insoluble analytically, and it is a common feature of population models that non-linearity renders the models insoluble analytically; it is then necessary to resort to numerical solutions using a computer. 


\begin{abstract}
Box 1. Simple Growth Models
Geometric and Exponential Growth

The geometric model is $N_{t+1}=\lambda N_{t}$. Here $N_{t}$ is the size of the population at time $t$, where $t$ is scaled to generations and $\lambda$ is the rate of increase each generation. In each generation the population size is $\lambda$ times the size it was in the previous generation. In this model, generations are discrete and nonoverlapping. This model is easy to solve. At any time $t, N_{t}=N_{0} \lambda^{t}$, where $N_{0}$ is the size of the population at time $t=0$. The exponential growth model is $d N / d t=r N$. The solution to this model is $N=N_{0} \exp (r t)$, where $\exp (r t)=e^{r t}, e$ being the base of natural logarithms, $r$ is the instantaneous rate of growth, and $N_{0}$ is the initial size of the population at time zero.

\section{Density-Dependent Growth}

With density-dependence, the geometric model becomes $N_{t+1}=\lambda N_{t} \exp \left(-g N_{t}\right)$, in which the exponential term has no real biological meaning, and is simply a convenient device to limit population numbers. The continuous version is the logistic equation: $d N / d t=r N(K-N) / K$, where $K$ is the carrying capacity imposed by resource limitation.
\end{abstract}

\title{
1.3. Host-Parasitoid and Predator-Prey Models
}

Modelling host-parasitoid and predator-prey systems in insect population dynamics has a long history, and the "workhorses" are the Nicholson-Bailey difference equation model and the Lotka-Volterra differential equation model (Box 2). Hassell (1978) described such models, and these models have been used in modelling sterile insect releases for populations under the influence of biotic interactions with other species.

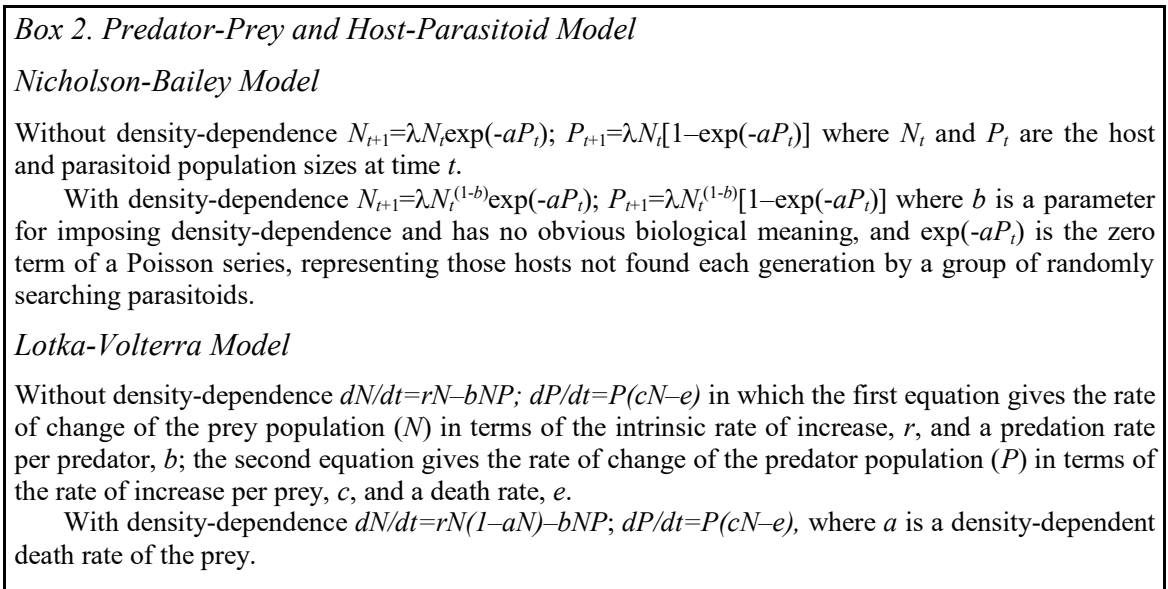

\section{MODELS OF STERILE INSECT RELEASES}

\subsection{Three Kinds of Control Programmes Using Sterility}

There are three methods of using sterile insects for population control. These are: (1) the standard method of releasing sterile males (or males and females) that have been 
reared and sterilized; earlier work in modelling of sterile releases was previously summarized by Hamada and Miyai (1985); (2) the treatment of insects with substerilizing doses of radiation or chemosterilants so that the matings are partially sterile, but the offspring of matings involving treated insects are sterile, called inherited sterility (Kean et al. 2007; Marec et al., this volume); and (3) the deployment of chemosterilants in field traps to sterilize insects that are attracted to the traps. It is mainly the first of these three methods that will be dealt with here. Chemosterilants are seldom used in the field because of their carcinogenic potential and the related risk of bioaccumulation in the food chain, although modelling has been done on this technique by Knipling (1960), Lawson (1967), Staley et al. (1971), Hawkes and Coaker (1977), Barclay (1981a), and Wall and Howard (1994). In addition, although the SIT is usually used for insect control, in some cases the concept can apply to other animals (Klassen et al. 2004).

\subsection{Initial Contribution of Knipling to Modelling the SIT}

Knipling produced a simple numerical model that foreshadowed most future modelling developments (Knipling 1955, 1959). The central feature of Knipling's model, and one found in almost all subsequent models, is the ratio of fertile males to all males in the population: $(M /(S+M))$ where $M$ is the number of fertile males (or females, assuming a 1:1 sex ratio) and $S$ is the number of sterile males. This gives the proportion of the population, under ideal conditions, that results in fertile egg production as a result of some fertile females mating with fertile males. Knipling's (1955) model for the release of sterile insects was a simple modification of the geometric model in Box 1 using the sterility factor above:

$$
F_{t+1}=\lambda F_{t}\left(M_{t} /\left(S+M_{t}\right)\right)
$$

where $F_{t}$ and $M_{t}$ are again the population size (fertile females and males) at time $t, \lambda$ is the rate of increase per generation, and $S$ is the release rate of sterile males each generation. This yields a stable steady state at $F=0$ and an unstable positive steady state for $F$ when $S=S^{*}$, the critical release rate, where $S^{*}=F(\lambda-1)$, the value of sterile release rate that holds the population at the steady state (Berryman 1967). If $S>S^{*}$, then the pest population will collapse and be eliminated. If $S<S^{*}$, then the population in this model will increase indefinitely.

\subsection{Sex Ratio}

One question asked early in work on releasing sterile insects was, "Is there an optimal sex ratio for the sterile insects being released?" It was initially thought that the release of sterile females would be counterproductive. This question was first addressed by Ailam and Galun (1967) and Lawson (1967); using probabilistic models of mating, they predicted that the release of females should never be detrimental (assuming they are all fully sterile), and in fact may assist the control programme if males are limited in their mating ability, in which case some fertile 
females might not get mated. However, for female-choice mating systems such as fruit flies the opposite was shown; in the absence of sterile females, sterile males are 3-4 times more effective because they focus only on fertile females and do not waste their limited sperm on sterile females (Hendrichs et al. 1995; Rendón et al. 2004). This was confirmed theoretically for female-choice mating systems, such as in tephritids (Vreysen et al. 2006). On the other hand, for moths (which have a very different mating system) the limited field evidence so far indicates that there is no advantage to male-only releases. In addition, for insects such as mosquitoes where females are disease vectors, it is essential not to release sterile females because that might increase the number of disease vectors.

\subsection{Residual Fertility of "Sterile Insects"}

If some of the treated insects are not completely sterilized, then the situation becomes more complicated. Klassen and Creech (1971) constructed a simple numerical model in which a certain proportion of the released males remained fertile. They found an upper limit to this "residual fertility" that was compatible with the success of the release programme. Their model can be put into algebraic form and generalized. When there is incomplete sterilization of the released insects, a fraction, $q$, of males remains fertile. In that case, Knipling's model can be modified as in Box 3. The critical sterile release rate is then only finite for $q<1 / \lambda$. If $q>1 / \lambda$, then the population is not controllable by sterile releases. Thus, for example, if the rate of increase, $\lambda$, is 10 , then $q$ must be less than 0.1 , i.e. the released males must be greater than $90 \%$ sterile in order for control by the SIT to be possible. Also, if the residual fertility is more than about three-fourths of the limiting value, then the required rate of sterile releases has to be much higher than with complete sterility (Fig. 1).

If both males and females are released and neither sex is completely sterile, then the fertile male $\mathrm{X}$ fertile female matings can be modelled as in Box 3. If residual fertility exists in both sexes following release, it becomes impossible to eliminate the pest population by sterile releases alone; the best that can be done is to suppress it to a low level with continuing sterile releases. In addition, control is impossible unless $q_{m}<F / \lambda\left(F+q_{f} S_{f}\right)$, where $q_{m}$ and $q_{f}$ are the residual fertilities of males and females, respectively. This value of $q_{m}$ is smaller than that without the release of females (Fig. 2). Thus, less residual male fertility can be tolerated with the release of residually fertile females (Barclay 2001). Fortunately, in most species targeted by the SIT, females are more sensitive than males to radiation sterilization (sperm are post-meiotic cells, whereas mature eggs are pre-meiotic), and therefore it is possible to identify sterilizing doses for the SIT that assure full female sterility (Bakri et al., this volume; Robinson, this volume).

\subsection{Competitive Ability of Males}

The ability of sterile males to compete with wild males for mates can be affected by sterilization through the debilitating effects on either sperm competition or the 
behaviour of the adults (Parker, Vreysen et al., this volume; Lance and McInnis, this volume). This problem was modelled by Berryman (1967), Bogyo et al. (1971), Berryman et al. (1973), Itô (1977), and Barclay (1982a). Their models are summarized by the model in Box 4 . All of their models show that the critical release rate increases as the competitive ability of sterilized insects decreases.

Box 3. Residual Fertility of "Sterile Insects"

Here Knipling's model becomes: $F_{t+1}=\lambda F_{t}\left(M_{t}+q S\right) /\left(M_{t}+S\right)$, where it is assumed that either only males $\left(M_{t}\right)$ are released or that released females are completely sterile and only males display residual fertility. This model has a stable steady state at $F=M=0$, and an unstable positive steady state for $F$ and $M$ when $S=S^{*}$, the critical release rate, where $S^{*}=M(\lambda-1) /(1-\lambda q)$. Here, $S^{*}$ is only finite for $q<1 / \lambda$.

If both males and females are released and neither sex is completely sterile, then the fertile male $\mathrm{X}$ fertile female matings can be modelled by the equation: $F_{t+1}=\lambda\left(F_{t}+q_{f} S_{f}\right)\left(M_{t}+q_{m} S_{m}\right) /\left(M_{t}+S_{m}\right)$, in which $F_{t}$ is the number of wild fertile females in generation $t, q_{m}$ and $q_{f}$ are the proportions of treated males and females, respectively, that remain fertile, and $S_{m}$ and $S_{f}$ are the number of treated males and females, respectively, that are released each generation (Barclay 2001). So far there is no restriction on the sex ratio. Thus $q_{f} S_{f}$ released females that remain fertile are added to the wild fertile females each generation, and $q_{m} S_{m}$ released males are added to the number of wild fertile males each generation, with the assumption that treated insects are equally competitive for mates with wild insects. This model has a lower stable steady state and an upper unstable steady state for $F$ and $M>0$ when $S=S^{*}$, and $S^{*}{ }_{m}=(\lambda$ $1)\left(F M+\lambda q_{f} S_{f} M\right) /\left(F\left(1-\lambda q_{m}\right)-\lambda q_{m} q_{f} S_{f}\right)$, and this equation is only soluble if the sex ratio is known. If we assume a one-to-one sex ratio (where $M_{t}=F_{t}$ and $S_{m}=S_{f}=S$ ), then we obtain a quadratic equation: $\lambda q_{m} q_{f} S^{2}-\left[1-\lambda\left(q_{m}+q_{f}\right)\right] F S+(\lambda-1) F^{2}=0$ which gives two roots when solved for either $S$ or $F$. The upper root of $F$ is unstable, and represents the size of the population before initiating sterile releases. The lower root of the equation for $F$ is stable, and is the value at which the population would be maintained by residual fertility after collapse due to suppression by sterile releases. Thus it is impossible to eliminate the pest population by sterile releases alone; also control is only possible if $q_{m}<F / \lambda\left(F+q_{f} S_{f}\right)$. The relationship between the maximum values of $q_{m}$ and $q_{f}$ is hyperbolic (Fig. 2).

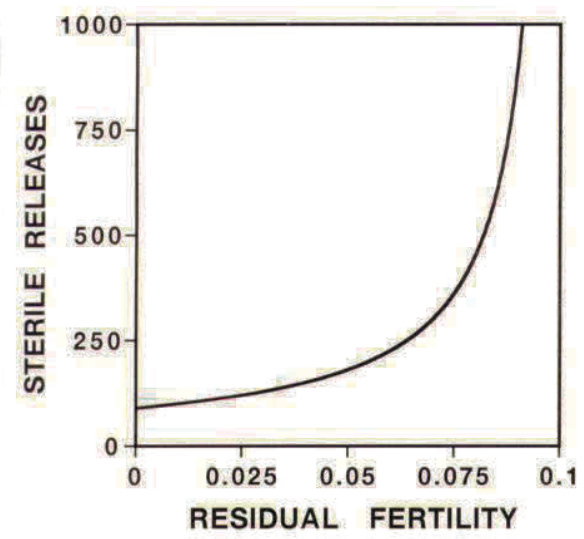

Figure 1. The critical sterile release rate, $S^{*}$, as a function of residual fertility, $q . \lambda=10$.

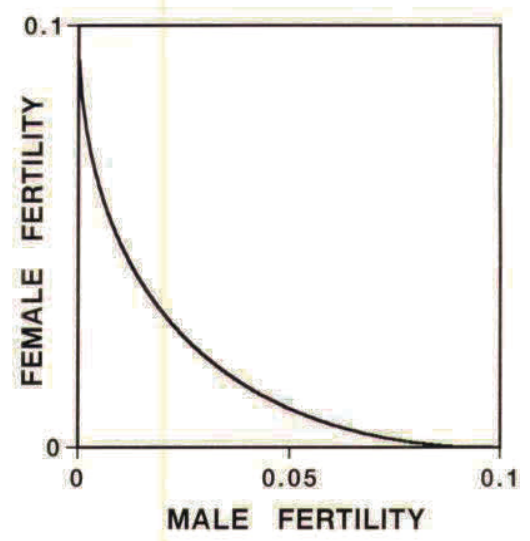

Figure 2. Allowable residual fertility when it is in both males and females. $\lambda=10$. 
Box 4. Competitive Ability of Males

We define $c$ as a coefficient of competitive ability, with 0 being completely non-competitive and 1 being fully competitive. Then $F_{t+1}=\lambda F_{t}\left(F_{t} /\left(F_{t}+c S\right)\right)$. This model has a stable steady state at $F=M=0$ when $S>0$. The positive (unstable) steady state for $F$ occurs when $S=S^{*}$, the critical value, where $S^{*}=(\lambda-1) F / c$, which is greater than $(\lambda-1) F$, with full competitive ability (Itô et al., this volume).

\subsection{Interactions of Residual Fertility and Competitiveness}

In this model only males display residual fertility; females are either completely sterilized or not released.

\subsubsection{Residually Fertile Insects Are Fully Competitive}

Here the insects that remain fertile after treatment are fully competitive with wild insects (Box 5). The allowable residual fertility of males is an almost linear function of the competitive ability of released sterile males (Fig. 3A, solid line), unless $\lambda$ is very small. Also, for a given degree of residual fertility, $S^{*}$ becomes larger as $c$ becomes smaller (Fig. 3B). Thus, the extent of residual fertility, and the degree of competitive ability that is lacking, tolerable to achieve some control, each have to be more restricted in the presence of the other (Barclay 2001). In this case, each of $c$ and $q$ will be more restrictive than is allowable if only one of these limitations exists (both must be within the range that allows for control by the SIT).

Box 5. Residual Fertility and Competitiveness
If residual fertile insects are fully competitive, $F_{t+1}=\lambda F_{t}\left(F_{t}+q S\right) /\left(F_{t}+q S+c S(1-q)\right)$, then this
model has a stable steady state at $F=M=0$ when $S>0$. The positive (unstable) steady state occurs
when $S=S^{*}=(\lambda-1) F /(c(1-q)-q(\lambda-1))$, and $S^{*}$ is finite only if $q<c /(\lambda-1+c)$.
If residual fertile insects are of reduced competitiveness, then the model becomes
$F_{t+1}=\lambda F_{t}\left(F_{t}+c q S\right) /\left(F_{t}+c S\right)$. If there is no residual fertility, there is a stable steady state at
$F=M=0$, if $S>0$, and a positive unstable steady state when $S=S^{*}=(\lambda-1) F / c$.
If there is both residual fertility and unequal male competitive ability, the steady state for $F$
is at $S^{*}=(\lambda-1) F /(c(1-q \lambda))$, and this is finite only if $q<1 / \lambda$.

\subsubsection{Residually Fertile Insects Have Reduced Competitiveness}

Here the insects that remain fertile after treatment are not fully competitive with wild insects (Box 5). The critical sterile release rate in this case (Fig. 3C and Box 5) is not as large as the corresponding value when residually fertile insects are of full competitive ability (Fig. 3B).

\subsection{Mating Patterns}

Another question asked early in the use of sterile releases was, "Should the females of the target species in a sterile release programme mate once or more than once?" The question has been addressed by Von Borstel and Knipling (1960) and Knipling (1964), and in the models of Berryman (1967), Lawson (1967), Zouros (1969), and 
Barclay (1984). The answer appears to be that female remating (polygamy) is quite compatible with the SIT, as long as mating is random, with sterilized male sperm being fully competitive with wild male sperm. In addition, in polygamous species, it doesn't matter whether sperm is diluted, replaced or excluded after the first mating, again as long as mating is random, and sterile males are fully competitive (Lance and McInnis, this volume).
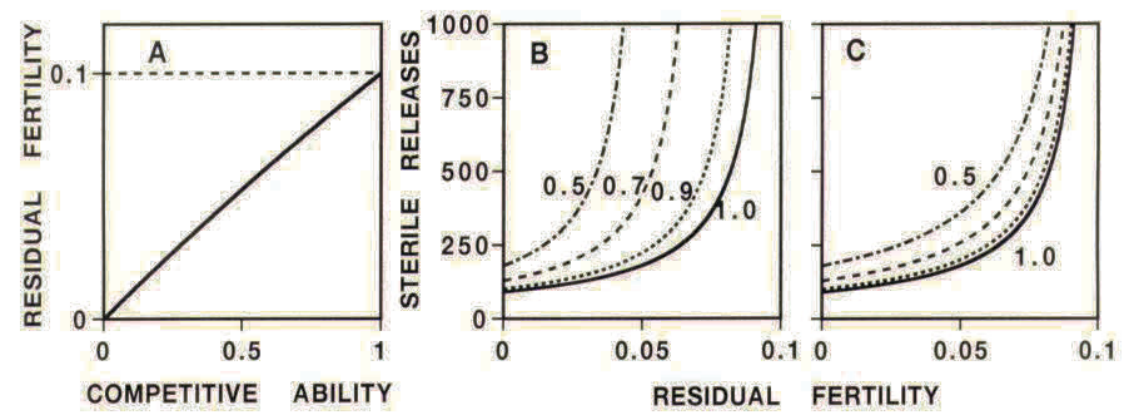

Figure 3. A: Changes in allowable residual fertility with changes in competitive ability of sterile males. Residually fertile insects are of reduced competitive ability (broken line) or are fully competitive (solid line). $B$ and $C$ : Critical sterile release rates for residual fertility ( 0 to

$0.1)$ and competitive ability $(0.5,0.7,0.9,1.0)$. In $B$, residually fertile insects are fully competitive, and in $C$, residually fertile insects are of reduced competitive ability. $\lambda=10$ in all cases. (Figure from Barclay 2001, reproduced with permission.)

\subsubsection{Interaction of Polygamy with Competitive Ability}

If sterile males are equally competitive for mates with fertile males, sterile sperm is fully competitive with fertile sperm, and there is no residual fertility, then the effects of polygamy (multiple female mating) are simply to reshuffle the sperm at each mating, and polygamy has essentially no effect. On the other hand, if sterilization is incomplete, and/or sterilized males (or their sperm) are of inferior competitive ability, then the situation is more complex. The work of Berryman (1967) (also addressed by Zouros (1969)) is particularly insightful in this matter. If only the first mating of a female results in sperm retention, or if all of the sperm of previous matings is replaced at each successive mating, and if all matings occur before oviposition begins, then the effective number of matings is just one. If the sperm from all matings mixes and is retained, then the effect of multiple matings depends on sperm competition as well as on competition between sterile and fertile males for mates. Berryman (1967) addressed this important problem, and it is worthwhile revisiting his results, making appropriate changes in his notation to make it consistent with the development above. Berryman considered three cases, depending on sperm action (Box 6). 


\begin{abstract}
Box 6. Mating Patterns
Interaction of Multiple Female Mating and Competitiveness

The probability of a fertile female mating with a sterile male is defined as $P_{s}=c_{a} S /\left(F_{t}+c_{a} S\right)$, and the probability of a fertile female mating with a fertile male as $P_{f}=F_{t} /\left(F_{t}+c_{a} S\right)=1-P_{s}$, where $c_{a}$ is the competitive ability of sterile adults (equivalent to $c$ in Box 4). Berryman (1967) considered the joint distribution of the number of matings, and the number of sterile matings, as a sequence of marginal distributions of the number of sterile matings given the number of matings. Thus a female can mate from zero to $M$ times, and for a given number, $m$, of matings the number of sterile matings is binomially distributed. If ${ }_{m} C_{n}$ is defined as the number of combinations of $m$ things taken $n$ at a time $(=m ! / n !(m-n) !)$, then the conditional probability that a given female mates with $n$ sterile males, given that she mates $m$ times, is $P(n \mid m)={ }_{m} C_{n} P_{s}^{n} P_{f}^{m-n}={ }_{m} C_{n} P_{s}^{n}\left(1-P_{s}\right)^{m-n}$. This is one term of a binomial distribution that describes the number of sterile matings given the number of matings, and there will be $M+1$ such distributions, including one for no matings. Berryman considered three cases, dependent on sperm action.

\section{Non-Functional Sperm}

If the sperm of sterilized adults is either nonexistent or immotile, then a female mating $m$ times will only produce sterile eggs if all the matings were with sterile males. The probability of this occurring is $P_{s}^{m}$, and so the probability of at least one fertile mating is $\left(1-P_{s}{ }^{m}\right)$. Then the probability of at least one fertile mating, over the range of mating frequencies, is $\Sigma P_{m}\left(1-P_{s}{ }^{m}\right)$ for $m=1,2,3, \ldots, M$, and so the population equation becomes $F_{t+1}=\lambda F_{t} \Sigma P_{m}\left(1-P_{s}^{m}\right)$ for $m=1,2,3, \ldots, M$, where $P_{m}$ is the probability of mating $m$ times.

\section{Dominant Lethal Mutations}

In the binomial expansion of the probabilities of $m$ matings, where $m$ goes from 0 to $M$, each of the terms representing mixed fertile and sterile matings will be weighted by a factor, $c_{s}$, representing the competitive ability of sterile sperm. Thus the probability of an egg being fertilized by a sterile sperm, taken over all mating frequencies, will be $P(e)=P_{s}{ }^{m}+c_{s}((m-1) / m)_{m} C_{m-1} P_{s}^{m-1}\left(1-P_{s}\right)+c_{s}((m-2) / m)_{m} C_{m-}$ ${ }_{2} P_{s}{ }^{m-2}\left(1-P_{s}\right)+\ldots .+(0 / m){ }_{m} C_{0}\left(1-P_{s}\right)^{m}$, which can be reduced to $P_{s}{ }^{m}+c_{s} P_{s}\left(1-P_{s}{ }^{m-1}\right)$, where $P_{s}=c_{a} S /\left(F_{t}+c_{a} S\right)$, as above. We can write the equation as $F_{t+1}=\lambda F_{t} \Sigma P_{m}\left[1-\left(P_{s}^{m}+c_{s} P_{s}\left(1-P_{s}^{m-1}\right)\right)\right]$ for $m=1,2,3, \ldots, M$. The values of the critical release rates, $S^{*}$, are shown in Fig. 5A and Fig. 5B against the adult sterile competitive ability, $c_{a}$, and for the sperm competitive ability, $c_{s}$ in Fig. $5 \mathrm{C}$. In addition, the values of $c_{a}$ and $c_{s}$ are shown for given values of the critical release rate $(250,500), S^{*}$, when it is held constant (Fig. 5C); as shown, there is a trade-off between $c_{a}$ and $c_{s}$.
\end{abstract}

\title{
2.7.2. Non-Functional Sperm
}

If the sperm of sterilized adults is either non-existent or immotile, then a female mating $m$ times will only produce sterile eggs if all the matings were with sterile males. The resulting critical values (Box 6) of the sterile release rate, $S^{*}$, are shown in Fig. 4 for several values of $M$, the maximum number of matings, values of the adult competition coefficient from 0.5 to 1.0 , two values of the probability of mating, and assuming a binomial distribution of mating frequencies.

\subsubsection{Dominant Lethal Mutations with Fully Competitive Sperm}

If sterility is caused by dominant lethal mutations, and the sperm of sterilized adults is fully competitive with that of fertile adults, then it can be shown that the probability that an egg is fertilized by a sterile sperm is independent of the number of matings, and the results from section 2.5 . on competitive ability still hold with polygamy, and correspond to the case of $M=1$ in Fig. 4. 

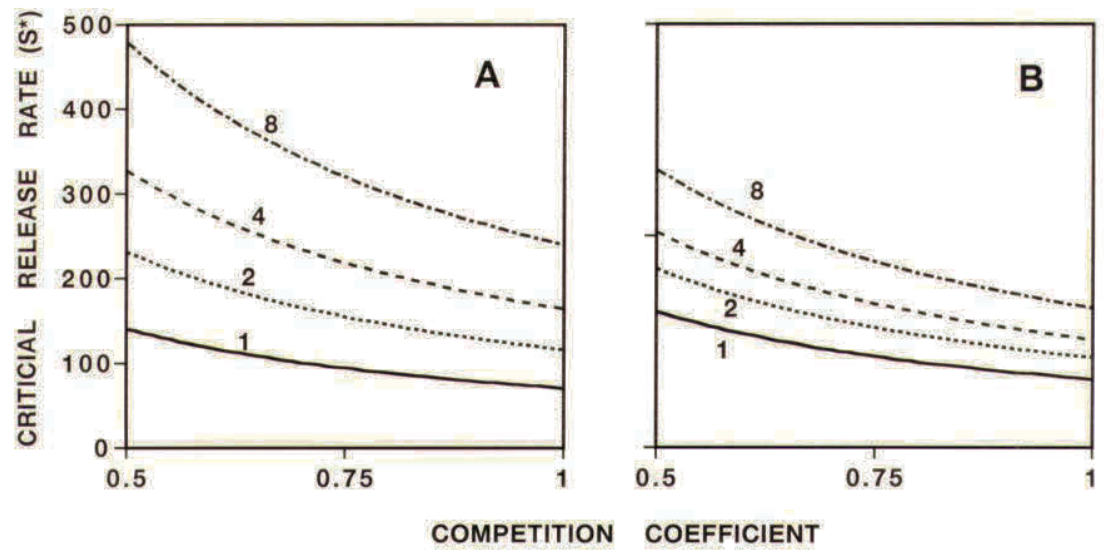

Figure 4. Critical sterile release rates when sterile males produce non-functional sperm. The maximum possible number of matings, $M$, is $1,2,4,8$, and the probability of mating in $A$ is 0.8 , and in $B$ is 0.9. The adult competition coefficient ranges from 0.5 to 1.0. $\lambda=10$ in all cases. (Figure generated from equations, Berryman 1967.)

\subsubsection{Dominant Lethal Mutations with Reduced Sperm Function}

If sterility is caused by dominant lethal mutations, and the sperm of sterilized adults is of reduced competitive ability compared with that of fertile adults, then it can be shown that the probability that an egg is fertilized by sterile sperm depends on the number of matings. The values of the critical release rates, $S^{*}$, in Box 6 , are shown in Fig. 5A and Fig. 5B against the adult sterile competitive ability, $c_{a}$, and in Fig. 5C for the sperm competitive ability, $c_{s}$. In addition, the values of $c_{a}$ and $c_{s}$ are shown for given values of the critical release rate $(250,500), S^{*}$, when the critical release rate is held constant (Fig. 5C); there is a trade-off between $c_{a}$ and $c_{s}$, the presence of each one limiting the value of the other.
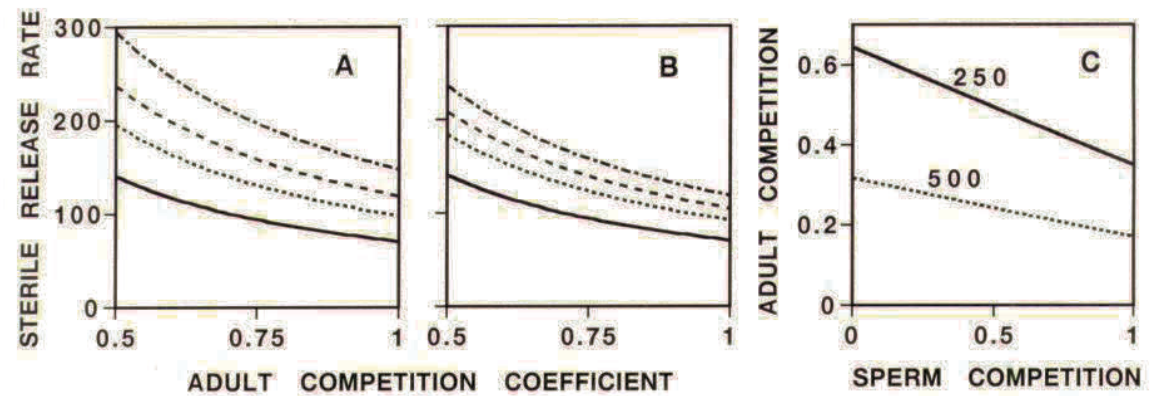

Figure 5. A and B: Values of the critical release rates, $S^{*}$, for values of adult sterile competitiveness, $c_{a}$, four mating frequencies $(1,2,4,8)$, and two probabilities of mating A: 0.6, B: 0.8. C: Limits on adult sterile competitiveness compatible with control of the pest population; these are shown for a range of values of sperm competitiveness and two values of sterile releases (250, 500). (Figure generated from equations, Berryman 1967.) 


\subsubsection{Choice of Mates}

Mating systems of insect pests amenable to SIT application can be categorized as (1) resource defence-based mating systems, (2) non-resource-based mating systems, and (3) lek and swarm mating systems (Hendrichs et al. 2002; Lance and McInnis, this volume). Systems (1) and (2) are mostly determined by male competition, and are male-choice mating systems. System (3) is a female-choice mating system, where the female chooses a mate from among the courting males in the lek. The distinction between male- and female-choice systems, as well as the propensity of wild individuals to mate with their wild counterparts rather than sterile insects, has implications for the effectiveness of an SIT project. Using mating models, Vreysen et al. (2006) examined these questions, as well as the release of only males or of both sexes, and arrived at the following conclusions.

In a male-choice mating system, such as in the New World screwworm Cochliomyia hominivorax (Coquerel), overcoming a situation where there is a propensity of wild males to preferentially mate with wild females if sterile females are also being released, would require a doubling of the number of sterile males compared with male-only releases. In the model on female choice, the release of both sexes and male-only releases required the same sterile to wild male overflooding ratio. This, however, is at odds with field projects that have shown a significant benefit with male-only releases against insects which have a femalechoice mating system, e.g. Mediterranean fruit fly Ceratitis capitata (Wiedemann) (Hendrichs et al. 1995; Rendón et al. 2004). Models were constructed to assess the potential effect of sterile female presence or absence on reduced sterile sperm quantity with remating, reduced sterile sperm quality with aging, and incomplete redistribution of the sterile males with the wild insects. In all three cases, male-only releases result in relatively more efficient sterile insects compared with projects releasing both sexes (Vreysen et al. 2006).

A problem with female-choice mating systems is that any deficiency in sterile male behaviour or function can bias females against mating with them, and this can have severe consequences for the success of any SIT project.

\subsection{Interaction of Polygamy with Residual Fertility}

A similar analysis on residual fertility (not shown) can be performed. Starting with the equation for residual fertility, and assuming that sterile sperm are fully functional, one proceeds as with the case of dominant lethal mutations and fully functional sperm. The result is that the probability that a female will mate with a sterile male is independent of the number of matings (Barclay 2001), and the probability that an egg will be fertilized by a sterile sperm is also independent of the number of matings. Thus, multiple female mating (polygamy) and residual fertility have no interaction, and the results derived above for residual fertility alone apply both to monogamy and polygamy. 


\subsection{Stochastic Models}

Stochastic models involve the specification of certain variables as being random. If the processes involved are well known and the extent of variation is known, then stochastic models can give additional information on the expected variability of the resulting control, as well as deviations of mean values from those predicted by deterministic models. This is especially true in areas like genetics in which the mechanisms of variation, e.g. meiosis, are clear. However this information is often not well known in animal ecology, and therefore stochastic models may be of limited use. In fact, if the wrong features are allowed to vary (e.g. birth rate is variable in the model, whereas in reality it might be mortality that varies), then stochastic models can give misleading results. In addition, unless they are solved numerically, stochastic models are usually much more difficult to analyse than deterministic models, and for these reasons the history of stochastic population modelling has been rather disappointing.

Stochastic models of sterile insect releases were developed by Kojima (1971), Bogyo (1975), Costello and Taylor (1975), Taylor (1976), and Kimanani and Odhiambo (1993), and they confirmed the results of Knipling (1955) and others that used deterministic models. They also derived a threshold release rate that leads to local extinction, and showed that much greater release rates above this threshold will not result in a greatly reduced time to extinction, although Lawson (1967) and Itô and Kawamoto (1979) offered evidence to the contrary using both a deterministic model and a probabilistic model.

\subsection{Population Movement}

The release of sterile insects, together with immigration from outside the control area, can be modelled by a simple modification of the model in Box 4 (Dietz 1976; Prout 1978). Flores (2003) has also provided a diffusion approach to the problem of immigration. Following Prout, two cases must be accommodated here: (1) immigration before mating, and (2) immigration after mating. In these two models it is assumed that all sterilized insects that are released are completely sterile.

\subsubsection{Immigration before Mating}

Assuming that $V$ males and $V$ females immigrate each generation prior to mating, the female immigrants are thus available for mating with the released sterile males as well as the wild males, and the male immigrants are available for competing with the sterile males. The model (Box 7) has two positive roots for $F$, with the upper one being unstable (the population as it existed just prior to sterile releases) and the lower one being stable. This lower steady state represents a population in a state of collapse due to sterile releases, but which is replenished each generation by immigrants. Note that zero is not a steady state solution here. The required sterile release rate grows rapidly with $V$, but there is no value of immigration that disallows control by sterile releases. The values of $S^{*}$ depend only modestly on $V$, if the immigration rate each generation is only a small proportion of the total population. 


\begin{abstract}
Box 7. Immigration
Immigration before Mating

If we include immigration into the model, we obtain $F_{t+1}=\lambda\left(F_{t}+V\right)\left(F_{t}+V\right) /\left(F_{t}+S+V\right)$. Solving for steady state, we obtain the quadratic: $(\lambda-1) F^{2}-[S-(2 \lambda-1) V] F+V^{2}=0$ which has two positive roots for $F$, with the upper one being unstable and the lower one being stable. Note that zero is not a steady state solution here.

The critical release rate is $S^{*}=[(\lambda-1) F+\lambda V](F+V) / F$

Immigration after Mating

The equation here is $F_{t+1}=\left[\lambda F_{t}^{2} /\left(F_{t}+S\right)\right]+\lambda V$. Note that, if the wild population is reduced to zero, it will be reconstituted the following generation, as then $F_{t+1}=\lambda V$. Again solving for steady state, we obtain $(\lambda-1) F^{2}-[S-\lambda V] F+\lambda V S=0$.

The critical sterile release rate is given by $S^{*}=F[(\lambda-1) F+\lambda V] /(F-\lambda V)$, and sterile releases can only control the population if $V<F / \lambda$.

For a given immigration rate, the required sterile release rate is much higher if immigration occurs after mating rather than before mating.
\end{abstract}

\title{
2.10.2. Immigration after Mating
}

In this case it is assumed that $V$ males and $V$ females immigrate each generation after mating. The female immigrants are thus not available for mating with the released sterile males or the wild males, however the male immigrants are available for competing with the sterile males. Thus, immigrating females remain fully fertile (unless there is some remating). Note that, if the wild population is reduced to zero, it will be reconstituted the following generation, as $F_{t+1}=\lambda V$. For a given value of the immigration rate, the required sterile release rate, $S^{*}$, is much higher if immigration is after mating than before mating, due to the fully fertile nature of the immigrating females.

\subsubsection{Combinations of Residual Fertility, Reduced Competitiveness, and Immigration}

Four models can be considered, being the four combinations of: (1) those sterilized insects that show residual fertility can be either of reduced competitive ability (reduced) or fully competitive (equal) with the wild insects, and (2) insects can immigrate either before or after mating.

Barclay (2001) provided equations for the four cases, and the values of the limiting residual fertilities are shown in Table 1 and Fig. 6. In Table 1, the allowable residual fertility for the case of residually fertile insects being of reduced competitive ability is the same as for the case involving sterile insects being fully competitive and immigration occurring. The other two cases yield more stringent limits on allowable residual fertility than with no immigration. It is apparent that there is a strong interaction among residual fertility, competitive ability, and immigration, with the feasible limits on each factor becoming much more restrictive in the presence of the other factors. 
Table 1. Limits on residual fertility $(q)$ when competitive ability of "sterilized but residually fertile insects" is either reduced or equal to that of wild fertile insects, and immigration is either before or after mating

\begin{tabular}{lll}
\hline Reduced Competitiveness & \multicolumn{1}{c}{ Equal Competitiveness } \\
\hline Before & $q<F / \lambda(F+V)$ & $q<c F[\lambda(F+V)-F(1-c)]$ \\
After & $q<(F-\lambda V) / \lambda F$ & $q<c(F-\lambda V)[\lambda F-(1-c)(F-\lambda V)]$ \\
\hline
\end{tabular}
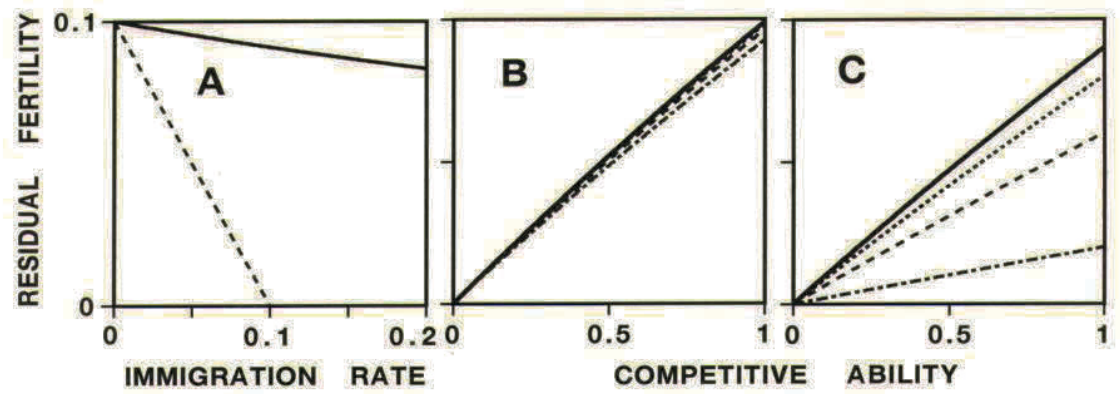

Figure 6. A: The allowable residual fertility $(q)$ when the released insects that are fertile are also fully competitive with wild insects. Immigration occurs before mating (_); immigration occurs after mating (- - - - ). B and C: Allowable residual fertility as a function of competitiveness of released insects. Immigration occurs at $1 \%($

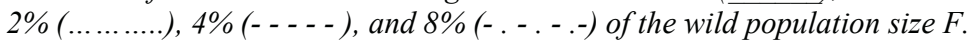

$B$ : Immigration occurs before mating. C: Immigration occurs after mating.

(Figure from Barclay 2001, reproduced with permission.)

\subsubsection{Large-Scale Population Movement}

The problem of large-scale population movement was addressed by Manoranjan and van den Driessche (1986), Lewis and van den Driessche (1993), Plant and Cunningham (1991), and Marsula and Wissel (1994). They showed, using diffusion equations, that dispersal of insects, coupled with non-linear growth terms, can result in waves of invasion or extinction. Both the velocity and direction of these waves depend critically on the rate of release of sterile insects. With low rates of release, the travelling wave advances as an invasion; when the density of sterile insects exceeds a critical density, the wave recedes, giving rise to local extinction. This is likely to have considerable relevance to programmes releasing sterile insects such as the New World screwworm eradication programme in the southern USA, Mexico and Central America, in which the pest population has been pushed back to Panama and a sterile insect buffer zone created. Matlock and associates are pursuing this approach with the screwworm, assessing the size of the buffer zone needed to ensure 
that insect invasion into the eradicated area does not occur (section 2.18.; R. B. Matlock, personal communication).

\subsubsection{Effects of Movement of Individuals on Efficiency of the SIT}

Apart from the effects of immigration by pest insects, the effect of diffusion (random non-directed movement) or redistribution of sterile insects within the control area appears to make control by SIT integration easier. Barclay and Vreysen (2013) presented a compartment model of tsetse (Glossina palpalis gambiensis Vanderplank) together with four control methods, one of which was the SIT. The critical sterile release rate for control increased with the degree of spatial aggregation but decreased with the extent of tsetse diffusion.

\subsubsection{Redistribution of Released Sterile Insects}

A problem that plagues many SIT projects is estimating the speed and extent of redistribution of sterile insects after aerial release, especially as sterilized insects may be less active and incur greater mortality than their wild counterparts (Parker, Vreysen et al., this volume; Dowell et al., this volume; Vreysen, this volume). Barclay et al. (2016) used diffusion equations to derive optimal spacing of flight lines and frequency of flights given the calculated sterile male population required to effectively cause population collapse. The optimization was done using a numerical treatment of the diffusion process to minimize total cost given the cost of sterile males and of flying time. They focussed on a midline between two flight lines of interest and calculated diffusive input of insects from eight flight lines on each side of the midline and from the eight previous flights. The optimization was found to depend strongly on the diffusion coefficient $(D)$ and also on the daily mortality $(\mu)$ of the released sterile males. Estimates of diffusion coefficients and daily mortalities were noted to vary widely among insect species, with $D$ being approximately 0.46 for tsetse flies and 0.006 for Mediterranean fruit flies, while $\mu$ varied between about 0.02 for tsetse and 0.23 for the fruit flies. At the low end of $\mu(0.04)$, longer flight intervals are suggested, and fewer sterile males need to be released; at the high end of $\mu(0.24)$, shorter flight intervals are suggested and higher numbers of sterile males need to be released.

These authors also derived a shorter approximate method of optimizing the sterile insect release based on the observation from the results of the numerical method that the optimal spacing of flight lines $(\omega)$ was twice the standard deviation, $2(2 D \tau)^{1 / 2}$, of the Gaussian distribution resulting from the diffusion process. Here $\tau$ is the time interval (days) between flights. Two assumptions of this method are: (1) the relationship between $\omega$ and the standard deviation of the distribution observed from the longer method is assumed to hold for other values of the parameters, and (2) the midline between flight lines being used for computations is assumed to be well within the control area so that edge effects are not evident and diffusion is assumed to be in equilibrium. This shorter method is outlined below. 
Given the values of $D$ and $\mu$, use each possible value of $\tau, \tau=1,2,3 \ldots . k$. For $n$ flights and a requirement of $N_{M}$ sterile males alive per $\mathrm{km}^{2}$, the number of sterile males to be released per $\mathrm{km}^{2}\left(N_{S K}\right)$ is:

$$
N_{S K}=N_{M} \frac{(\exp (\mu \tau)-1)}{}(1-\exp (-n \mu \tau))
$$

Now, to find the optimal value of $\tau$, we must minimize the formula for total $\operatorname{cost}\left(C_{T}\right)$ per $\mathrm{km}^{2}$ per day: cost of flying time + cost of sterile males:

$$
C_{T}=\mathrm{C}_{H} /(S \omega \tau)+\left(C_{S} / 10^{6}\right) N_{M}(\exp (-\mu \tau)-1) / \tau(1-\exp (-n \mu \tau))
$$

Here $C_{H}$ is the cost per hour of flying time, $S$ is the speed of the aircraft, and $C S$ is the cost per million sterile males. In the example below, $n$ is taken as 8 and $N_{M}$ is $10^{6}, C_{S}$ is USD 250 , and $C_{H}$ is USD $5 / \mathrm{km}$ actually flown over the field, once the transit to and from the field is included.

For Mediterranean fruit flies, $D=0.005$, so with $n=8$ flights, using these dollar figures,

$C_{T}(\tau)=5 /\left(2(2 D)^{0.5} \tau^{1.5}\right)+250 *(\exp (\mu \tau)-1) /(\tau(1-\exp (-n \mu \tau)))$

When $C_{T}(\tau)$ is plotted against $\tau$, this is a convex curve, i.e. a concave-up curve, with a minimum value that represents the optimal values of $\tau$ (Fig. 7). A hand-held graphing calculator, or graph-plotting software on a computer, can be used to obtain easily the minimum value, and the $\tau$ (which must be a positive integer) at which it occurs. When the cost starts to increase, one should stop calculating; the cost will only increase beyond this point.

As an example of the approximate method for $\mu=0.24$ using a basic calculator, we obtain the following: from Fig. 7, the best value of $\tau$ is 2 days. This then gives $\omega=2$ sqrt $(2 * 0.005 * 2)=0.283(=283 \mathrm{~m})$. The number of sterile insects to be released per day is then $1000000 \cdot(\exp (0.48)-1) /(2 *(1-\exp (-3.84)))=315000$.

\subsubsection{A Model for Eldana saccharina with Diffusion and Partial Sterility}

Potgieter et al. (2013) presented a stage-structured diffusion model for the African sugar cane borer Eldana saccharina Walker. Eleven stages were tallied. The released female moths were fully sterile while released males were partially sterile, and sterility existed in the $F_{1}$ generation. The habitat was heterogeneous and compartmentalized into relatively homogeneous subpopulations; diffusion provided the only movement among these subpopulations. The model was applied to an existing sugar cane field in KwaZulu-Natal in South Africa. Numerical results indicated that the optimal release distribution and release ratio depend on the dispersal capability of the insect, method of release, frequency of releases, and growth or mortality rate of the population (Potgieter et al. 2013). 


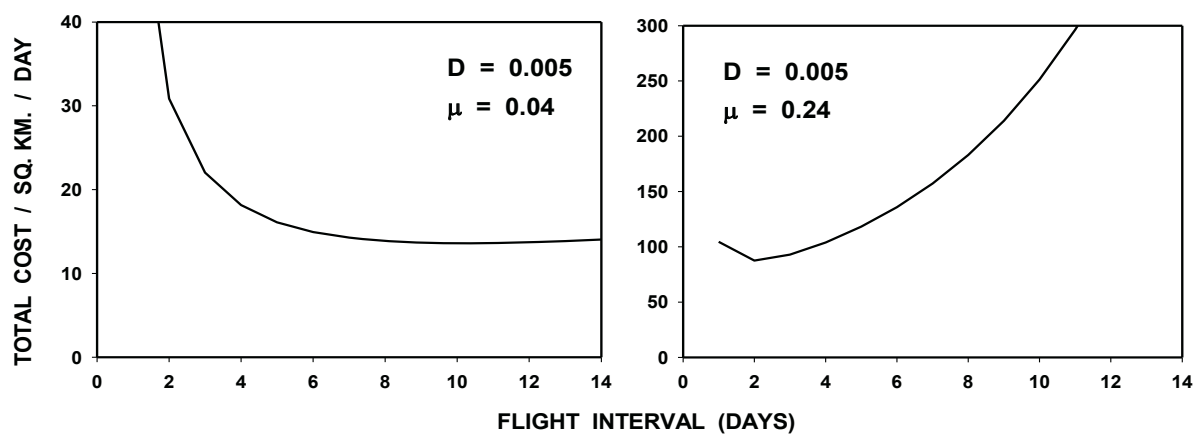

Figure 7. Curves for optimizing $\tau$ (the time interval (days) between flights) using the approximation. In both curves the value of $D$ is 0.005 , while in the left curve $\mu=$ 0.04 and in the right curve $\mu=0.24$. In both cases, 8 flights have been used. The location of the optimum value of $\tau$ is well defined $(\tau=2)$ for $\mu=0.24$, but not so well defined $(\tau=10)$ for $\mu=0.04$. For $\mu=0.24$ the cost would be notably greater if $\tau$ were assigned to 1 or 3 , whereas for $\mu=0.04$ the difference in cost would be minimal if $\tau$ was misassigned to be 8, 9, 11 or 12 when the optimum was 10.

\subsection{Density-Dependence in Population Regulation}

Density-dependence in a population has been shown by modelling to predispose it to control by sterile releases (Miller and Weidhaas 1974; Itô 1977; Prout 1978; Barclay and Mackauer 1980a). There are several formulations that include densitydependence in a model of a population, and all yield the same qualitative results. The two distinct ways that density-dependence assists the SIT are: (1) it reduces the effective biotic potential of a species by increasing the natural mortality at higher densities, and (2) it introduces a bifurcation (splitting of one root of the equation into two) into the model whereby the population suddenly collapses as the sterile insect release rate is increased above the level required at the bifurcation (Fig. 8). This avoids the necessity of the high levels of release needed to reduce the population below the unstable steady state in the model with no density-dependence. This bifurcation occurs in all the models involving density-dependence, and appears to result from the interaction of the depressing effects of density-dependence and the unstable equilibrium created by the SIT formulation in section 2.2., which results in the release of sterile insects being more effective at low density than at high population density, and thus the efficiency of the SIT increases as the population declines. The sudden collapse of a population under attack by sterile insect releases has indeed been observed in the programme against the melon fly Zeugodacus cucurbitae (Coquillett) in Okinawa, Japan (Iwahashi 1977). Thus, this predicted bifurcation appears to be a robust result, and one that apparently mimics nature.

The exact behaviour of the SIT under density-dependence appears to depend sensitively on the biology of the system. Lawson (1967) and Berryman et al. (1973) pointed out that overcrowded populations may deplete their resources sufficiently such that survival to the adult stage is low. In this case, killing some of them (or 
lowering egg production) might actually result in a higher survival to the adult stage, making the use of the SIT counterproductive in such a situation. Another situation might be encountered in the case of an insect species wherein egg production is much higher than the resource allows, e.g. the olive fruit fly Bactrocera oleae (Rossi), in which one egg per fruit is laid. If sterile eggs did not deter insects from laying further eggs in a fruit already containing a sterile egg, then reduction in fertile egg production would have to be substantial before any effect would be noticed.

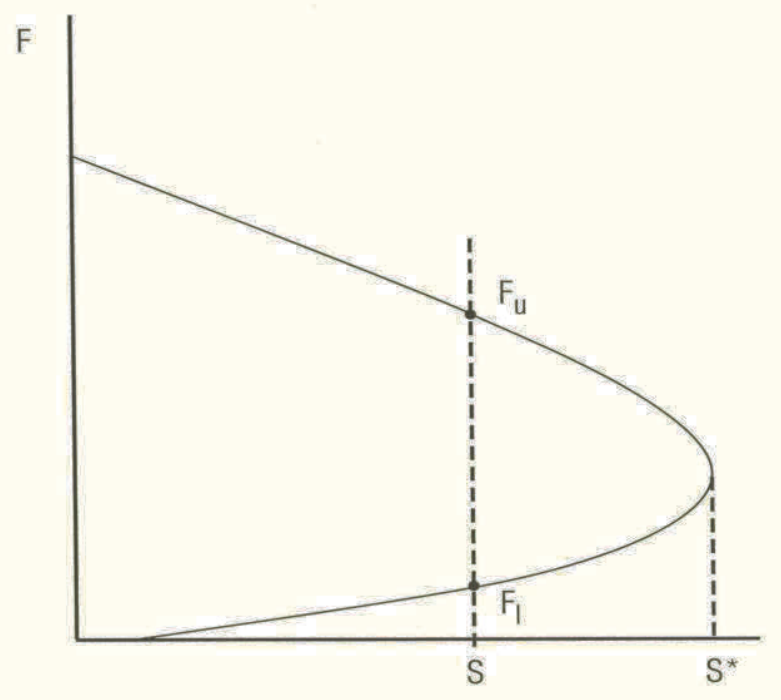

Figure 8. Isoclines formed by setting the equations for sterile insects (S, vertical line) and fertile insects $(F)$ to zero; where they cross are the two steady states. The upper one is stable, and the lower one unstable. As sterile releases increase, the sterile isocline moves to the right. The bifurcation is at the point where the isoclines are tangent to each other.

Larger values of sterile insect releases result in a sudden collapse of the population.

(Figure generated from equation 12, Barclay 2001.)

\subsection{Age Structure}

\subsubsection{Two Age Classes}

The existence of two or more life stages of a species complicates the dynamic responses of a population to mortality factors, especially if the two stages are ecologically different, as they are in mosquitoes and other pest species in which the two active stages occupy different habitats. If density-dependence is strong in one stage and weak or absent in the other stage, then the density-dependent stage is strongly buffered against mortality factors and tends not to vary greatly, while the other stage may vary more but the mean size is a linear function of the buffered stage. As such, somewhat different responses to mortality affecting mainly one stage 
would be expected, and indeed this appears to be the case. In the case of the SIT, the sterile insects released always affect the adult stage, reducing fertile egg production.

Prout (1978) modelled the SIT for species with identifiable age structure and subject to immigration. His results indicated that, if the larval stage caused the pest problem, a higher level of immigration of mated adults was tolerable.

Barclay (1980b) showed that the critical release rate $\left(S^{*}\right)$ is a larger proportion of the larval equilibrium size when density-dependence is in the larval survivorship than when density-dependence is in the adult survivorship. Thus, relative to a given larval equilibrium, the population requires fewer sterile insect releases when densitydependence is in the adult stage.

\subsubsection{Daily Age Classes}

Barclay et al. (2014) modelled a population of a monogamous species with egg, larval, pupal and adult stages, and a daily time step. Survivorship was age-specific, and there was no density-dependent population regulation. Eggs take ke days from oviposition to hatch, and there is no daily mortality. The numbers of eggs in the $i^{\text {th }}$ age class at time $t$ is $E_{i, t}$, and as there is no egg mortality, $E_{i+1, t+l}=E_{i, t}$ for $1 \leq i<k e$. Eggs of age $k e$ hatch into larvae, so that $L_{1, t+1}=E_{k e, t}$.

Larvae require $k l$ days for development, and the number of larvae in the $i^{\text {th }}$ larval age class at time $t$ is $L_{i, t}$ for $1<i \leq k l$. Larvae survive from age $i$ to age $i+1$ at a rate $q_{i}$, so that $L_{i+1, t+1}=q_{i} L_{i, t}$ for $1 \leq i<k l$. Similarly, pupae require $k p$ days for development, and the number of pupae in the $i^{\text {th }}$ pupal age class at time $t$ is $P_{i, t}$ for $1 \leq i \leq k p$. Pupae survive from age $i$ to age $i+1$ at a rate $u_{i}$, so that $P_{i+1, t+1}=u_{i} P_{i, t}$ for $1 \leq i<k p$. Also, the first pupal age class is $P_{1, t+1}=q_{k l} L_{k l, t}$.

There are five categories of adults, also referred to as adult population components; at age $i$ and at time $t$ they are: virgin females $\left(V_{i, t}\right)$, females mated to fertile (wild) males $\left(F_{i, t}\right)$, females mated to sterile males $\left(G_{i, t}\right)$, wild males $\left(M_{i, t}\right)$ and sterile males $\left(N_{i, t}\right)$. The virgin adult females are assumed to become receptive to male mating advances at age $\tau$ days, and all of age $\tau$ mate on that day and immediately start ovipositing. Adult virgin females, fertile-mated females and sterile-mated females all survive at a rate $w_{i}$ from female age $i$ to age $i+1$, except that mated females start on their day 1 at survivorship $w_{1+\tau}$ because adult emergence occurred $\tau$ days before. Wild males survive at a proportional rate $\mathrm{y}_{\mathrm{i}}$ from age $i$ to $i+1$, and sterile males correspondingly survive at a rate $\mathrm{z}_{\mathrm{i}}$. Thus, age classes $i$ at time $t$ survive as follows: $V_{i+1, t+1}=w_{i} V_{i, t} ; F_{i+1, t+1}=w_{i+\tau} F_{i, t} ; G_{i+1, t+1}=w_{i+\tau} G_{i, t} ; M_{i+1, t+1}$ $=y_{i} M_{i, t} ; N_{i+1, t+1}=z_{i} N_{i, t}$.

If sterile males are being released, then there is a release rate, $r^{*}$, at which the population will not increase further and a steady state (equilibrium) will be attained. This equilibrium is unstable in the absence of density-dependent regulation, and it is of interest only inasmuch as it separates success (if the actual release rate, $r$, is greater than $r^{*}$ ) from failure (if the sterile release rate is less than $r^{*}$ ). This sterile male release rate, $r^{*}$, is called the critical release rate, and it is what is calculated in standard models of the SIT, e.g. Berryman 1967; Dietz 1976; Itô 1977; Barclay and Mackauer 1980; Barclay 2001; Barclay et al. 2014).

Two derived statistics are required to calculate fertility, and thus to calculate the size of the first egg age class, and also to determine the critical release rate; these are 
the net survivorship from eggs to adult emergence, $\gamma$, and the mean daily fertility (mdf), $\mu$ (called "mean fertile eggs per fly-day" by Carey 1989):

$$
\begin{gathered}
\gamma=\left(\Pi_{1}^{(k l-1)} q_{i}\right)\left(\Pi_{1}^{(k p-l)} u_{i}\right) \\
\mu=\Sigma l_{x} m_{x} h_{x} / \Sigma l_{x}
\end{gathered}
$$

where $l_{x}, m_{x}$ and $h_{x}$ are the survivorship and fecundity of fertile-mated females and hatchability of their eggs, respectively, from age $x$ to age $x+1$ (Carey 1989); here $\mu$ (fertility) is not the same as $\mu$ in section 2.10.6. (mortality). The equation for the critical sterile release rate, $r^{*}$ (Barclay et al. 2014), is then obtained by solving the five adult equations at equilibrium simultaneously for $r$ in terms of $\mathrm{Fe}$, the fertilemated female subpopulation. Doing the appropriate substitutions and simplifying yields the critical value of $r, r^{*}$ :

$$
r^{*}=\gamma \mu F e a(b c-1) / d
$$

where:

$a=\left[\Sigma_{0}{ }^{(k m-1)} \prod_{0}{ }^{(i)} y_{j}\right]$, i.e. the sum of the products of fertile male survivorships;

$b=\gamma \mu\left[\Sigma_{0}^{(k f-1)} \Pi_{0}^{(i)} w_{j}\right]$, i.e. $\gamma \mu$ times the sum of the products of female survivorships;

$\left.c=\prod_{0}{ }^{(\tau)} w_{j}\right)$, i.e. the survivorship of virgin females to the $\tau^{\text {th }}$ age class; and

$d=\left[\Sigma_{0}{ }^{(k m-1)} \Pi_{0}{ }^{(i)} z_{j}\right]$, i.e. the sum of the products of sterile male survivorships.

Even though this appears to be rather complicated, it is easily computed if the agespecific fecundities, hatchabilities, and survivorships are known or can be estimated. Also, it is remarkably parallel to the models with no age structure, in which $\gamma \mu$ is the analogue of $\lambda$ in Knipling's model.

One further complication in calculating fertility should be mentioned. For the special case of species giving single live births of larvae at intervals of several days, such as tsetse flies, it has been common practice to calculate fertility as the reciprocal of the inter-larval period. It has been shown recently (Barclay et al. 2020) using eq. (6) above that a better estimate of fertility is about $10 \%$ greater than the reciprocal of the inter-larval period. This has implications for calculating the minimum sterile-male release rate required for control or eradication.

\subsubsection{Overflooding Ratios and Release Ratios}

The overflooding ratio, a key parameter, has long been a measure of the ratio of sterile males to wild males in the population (Steiner et al. 1965). It is sometimes thought of as the size of sterile releases compared with the wild population; this is approximately valid in species with short reproductive seasons and non-overlapping generations. However, in species with a long reproductive period, often extending throughout the warm season in temperate climates, a surviving population of sterile males exists following several releases, and the overflooding ratio is not equal to the periodic release rate divided by the wild male population size, and must be estimated by sampling or some other means. The overflooding ratio is here called $\varphi$. 
Another ratio is the release ratio, defined as the ratio between the periodic sterile male releases and the wild male subpopulation, and here called $\rho$. For species with one short reproductive period each year, the two $(\varphi$ and $\rho)$ are approximately equal, allowing for some mortality following release. Such populations yield the same sterile release rate required to stop population growth, and then hold the population at equilibrium, as that predicted by the model at equilibrium. In freely growing populations with overlapping generations, the release ratio and the overflooding ratio are not equal.

\subsubsection{Stopping a Growing Population}

Barclay et al. $(2014,2016)$ derived critical control rates that are required to hold an age-structured population at an unstable equilibrium. This equilibrium separates success (if the control rate is above the critical rate) from failure (if the control rate is below the critical rate). The paper by Barclay (2016) builds on a previous publication (Barclay et al. 2014) on the control of age-structured populations, and shows that the equilibrium statistics derived are only a first step towards being useful. They are not sufficient to allow calculation of the appropriate values of the sterile release rates and overflooding ratios required to stop a growing age-structured population using the SIT. A measure called the "required sterile release rate", $r$ ', is defined as the value of the sterile male release rate, $r$, necessary to stop a population growing freely in a stable-age distribution, i.e. with sterile-male releases, the growth rate eventually becomes zero with $r=r$ '. Such populations are largely undergoing density-independent growth, as may be the case with many spot infestations.

The values of $r$ ' were all moderately close to the calculated value of $r^{*}$, the critical rate, but the computed overflooding ratios were very different for a growing age-structured population than for one at equilibrium; the value of the overflooding ratio calculated for an equilibrium population, i.e. when $r=r^{*}$, greatly underestimates the value required to stop a population in unrestricted growth. As the value of $r$ ' increases with population size, accurate estimation of population size is necessary to calculate realistic values of $r$ '. For the Mediterranean fruit fly example that was developed (Barclay 2016), the discrepancy was huge; the overflooding ratio calculated from an equilibrium population was only about $1 \%$ that found to be required in the simulation. This is because, at equilibrium, females mated to fertile males constitute a very small part of the equilibrium population, whereas in a growing population, fertilized females constitute a much larger portion of the total population. Thus, in this situation, it is the actual critical release rate, $\mathrm{r}^{*}$, that should guide the control programme, rather than deriving the overflooding ratio from sampling the males in a freely growing population.

\subsection{Population Aggregation}

In nature most populations are not dispersed evenly over the available habitat. Some processes, such as territoriality, result in dispersion patterns that are more regular (Fig. 9, upper left) than one would expect of a random spatial distribution (Fig. 9, upper right). However, most populations will tend to have a somewhat aggregated 
dispersion pattern. Aggregation is the most difficult pattern to deal with in making sterile insect releases, because one has to know where the clumps are located (Fig. 9, lower panels).

Modelling of spatial aggregation has been done by Wehrhahn (1973) and Barclay (1992a). Wehrhahn used a mosaic of patches, inhabited by differing numbers of insects, and compared the required release rates for various patterns of aggregation. He used Monte-Carlo simulation, which introduces random numbers to allow stochastic variation, in this case, of migration rates among patches. Wehrhahn pointed out that the control programme itself will probably change the nature of the spatial distribution. Bakhoum et al. (2021) described using species distribution modelling and landscape genetics for tsetse fly control.
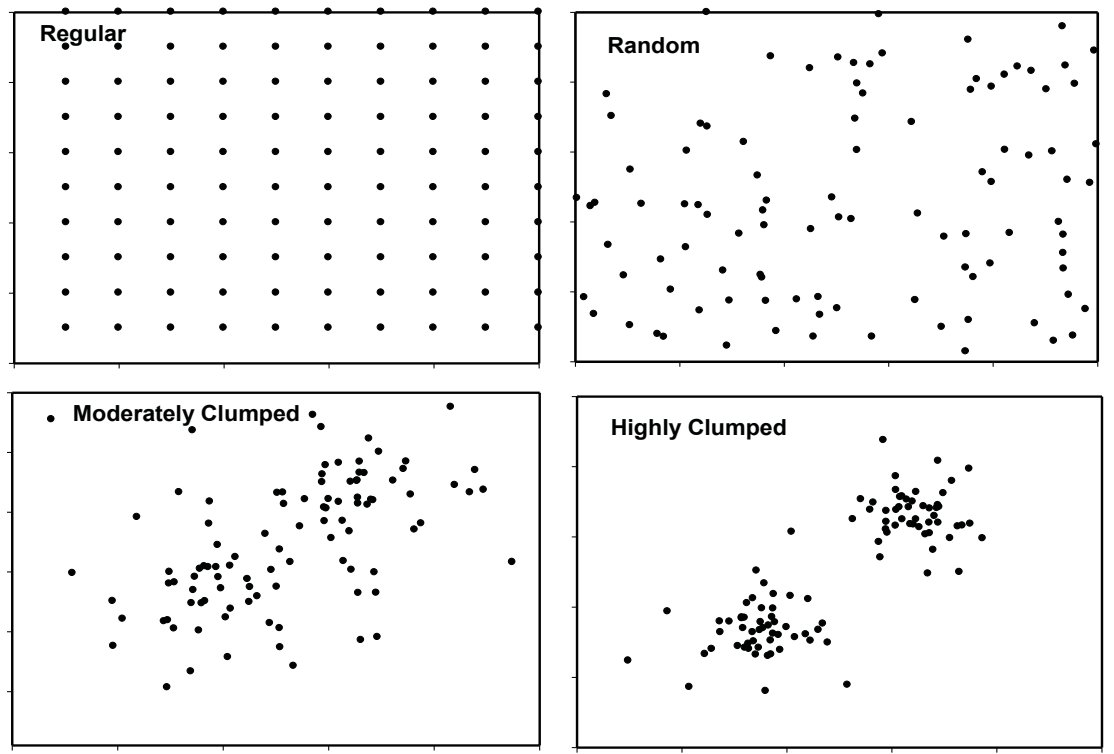

Figure 9. Examples of spatial distributions: regular (uniform), upper left; random, upper right; moderately clumped, lower left; highly clumped, lower right (reproduced from FAO/IAEA 2016). These can be quantified by various aggregation indices (Pielou 1969; FAO/IAEA 2016).

Another approach has used probability distributions to describe the extent of aggregation (Barclay 1992a). There is a long history of using these distributions in ecology, summarized by Pielou (1969) and Patil and Stiteler (1974). The most commonly used distribution to quantify aggregation is the Negative Binomial Distribution in which the parameter $k$ measures clumping. If aggregation is extreme, then $k$ is close to zero; as $k$ goes to infinity, the dispersion approaches a random pattern. Another approach uses $1 / k$, which increases with the degree of clumping. Barclay (1992a) used the negative binomial distribution to derive required sterile insect release rates of an aggregated population as a function of the clumping 
parameter, $k$. For moderately aggregated populations $(k=0.25)$, it was found that the required release rate was about four times that for a randomly dispersed population. Shiga (1986) analysed spatial distributions in the context of fruit fly eradication using male annihilation and the SIT.

Many aspects of aggregation involve behavioural components. Horng and Plant (1992) modelled the impact of lek mating on the SIT, using a Poisson binomial distribution. They found that the sterility effect, presence or absence of female matechoice, and sterile male mating competitiveness were the most important factors in their model in determining the success of a programme releasing sterile insects.

\subsection{Predation, Parasitism, and Competition}

The effects of predation on the efficiency of the SIT were first modelled by Knipling (1979) using a simple numerical model. His model predicted a synergistic interaction between predation and sterile insect releases, such that the net effect would be considerably greater than either alone. This would only be expected to be true if predation is random, with sterile and wild males equally able to evade predator attacks. Nevertheless, there is growing evidence that sterile males have largely lost this capacity and suffer much more predation (Hendrichs et al. 1994; Hendrichs and Hendrichs 1998; Hendrichs et al. 2007). On the other hand, the integration of augmentative parasitoid releases and inherited sterility can be highly synergistic (Barclay 1987a, b; Carpenter 2013).

Barclay and Mackauer (1980b) included sterile insect releases in the LotkaVolterra predator-prey model, and demonstrated that, not only was the critical release rate lower with than without predators, the system was also greatly destabilized, and population collapse often occurred with release rates well below the critical value. This model was subsequently shown by Harrison et al. (1982) to have a very complicated dynamic behaviour, and this is presumably related to the inherent instability. This model was then extended, and an even greater array of dynamical behaviour was found (Barclay and van den Driessche 1990). In addition, the general features of the predator-prey system were found similar to the situation involving hosts and parasitoids (Barclay 1987a). Knipling (1998) analysed extensively the effects of augmentation of predators and parasitoids on the efficiency of the SIT.

If the pest species is in competition for resources with another species, then this is of some value to the release programme, because it reduces the initial pest population size, but apart from that there seems to be little effect of the competing species on the release programme (Barclay 1981b).

\subsection{Stability under Various Conditions}

Stability is a very important aspect of populations, and is of special interest to pest managers. If, for any reason, the population is likely to collapse, this is important. Barclay (1982b) examined four systems or cases for their relative stability under sterile insect releases. These were all differential-equation models and represented: (1) a simple one-species model with only one identifiable life stage (adult), (2) a 
one-species model with two life stages (larval and adult), (3) a model of two competing species, one of which is the pest, and (4) a predator-prey model in which the prey is the pest. There are many possible definitions of stability in ecology, and they involve various dynamic characteristics of the system. None has yet emerged as definitive, although an extensive analysis of the topic is now available (Mueller and Joshi 2000). Barclay (1982b) examined five criteria of stability for each system, and ranked the systems. These stability criteria involved characteristics such as extinctions, time to extinction, amplitude of fluctuations, and time until return to equilibrium. The most stable was the single species - single stage model, followed by the competing species model, then the two-stage model, and by far the least stable was the predator-prey system above (section 2.14.). The existence of obligate predators (or parasitoids) both lowers the critical release rate of sterile insects and also destabilizes the system, so that it is likely to collapse even when the sterile release rate is lower than the critical rate. Unfortunately, there appears to be no experimental evidence to test these ideas.

\subsection{Integration of Control Methods}

Since the SIT works best at low pest densities (section 2.11.), it is common practice to reduce the population with an insecticide or other control method (or to start at naturally low population levels), prior to the release of sterile insects. This brings the population down to a level at which the number of sterile insects (required to be produced by a rearing facility) is manageable. It might also be possible to combine contemporaneously the action of the SIT with other control methods to share the required mortality among two or more imposed sources, each one then having to impose only a modest level of mortality, and each one perhaps operating best under conditions not favourable to the others (Barclay 1992b; Mangan and Bouyer, this volume). On the other hand, certain combinations might interfere with each other and thus prove unsuitable (Barclay 1987c).

Knipling (1964, 1979) examined several combinations of various control methods with the SIT, using simple numerical intra-generational models. These include combinations of sterile releases with insecticides, sterilants, pheromones, parasitoids or predators. Barclay $(1980 \mathrm{~b}, 1987 \mathrm{a}, \mathrm{b})$ and Barclay and van den Driessche (1989) also examined some of these combinations using more general inter-generational algebraic models. They found that the results of the combined use of sterile insects and other control methods became less clear when other biotic interactions were included; for example, a three-way interaction with sterile insects, predators, and insecticides may be counterproductive because the insecticides would probably reduce the effectiveness of the predators. In general, in population dynamics, three-way interactions are much more difficult to predict and analyse than two-way interactions.

\subsubsection{SIT with Application of Insecticide}

It might be thought that insecticides and the SIT are incompatible because the insecticide would kill sterile, as well as fertile, insects. However, Knipling (1964, 
1979) reasoned that insecticide application would kill both sterile and fertile insects in the same proportion, and thus maintain the overflooding ratio, rendering the two control methods compatible. By numerical examples he showed that these two methods could work well together, and thus reduce both total costs and the need for excessive insecticide application. If the sterile insects were also resistant to insecticide, then the combination would be even more effective.

Barclay $(1980 \mathrm{a}, \mathrm{b})$ found that, when the pest species was considered in isolation, the application of insecticide coupled with the release of sterile insects increased total mortality. However, the results of the combined use of insecticide and sterile insects became less clear when specific tritrophic biotic interactions were included, e.g. predators or parasitoids. For example, if the pest is already under considerable predation, the combination of insecticide and sterile insects might be detrimental. Therefore, when using sterile insects against species with two life stages, a larvicide appears to be more useful than an adulticide.

\subsubsection{SIT with Pheromone Traps for Male Annihilation}

Knipling (1979) found that the combined use of sterile releases and pheromone traps was less efficient than an equivalent effort put into either method alone. This was because of the interference caused by the killing of sterile males in the pheromone traps. As a variant of this combination, Knipling proposed that releasing pheromonetreated sterile insects could enhance mate-finding, thus increasing the competitive ability of sterile insects, especially at low densities. Knipling found that, for insects in which the males produce female-attracting pheromone, such as the boll weevil Anthonomus grandis grandis Boheman, the release of pheromone-treated males would substantially increase the effectiveness of the control programme, assuming that the applied pheromone did not deteriorate badly. Knipling also considered the situation in which females produce male-attracting pheromone, and he modelled the release of pheromone-treated sterile females alone. These would probably be most effective if they were free-living rather than contained in traps. He again found that this method was much more effective than the use of untreated sterile females, and that control might be possible using pheromone-treated sterile females where the release of only non-treated sterile females would be hopelessly inadequate. Pheromone-treated sterile insects of a species that is easier to mass-rear might also act as vehicles for mating disruption of the wild population of a different target pest (Suckling et al. 2011).

Hamada and Miyai (1985), using a continuous model, modelled the combination of the simultaneous release of sterile insects and pheromone trapping for male annihilation (Box 8). They found that the two methods in combination required less effort for each control method than when using either method alone. Their recommendation was to use male annihilation first and then sterile releases. Although the model did not specifically explore that scenario, this approach was successfully applied in Japan against the melon fly and the oriental fruit fly Bactrocera dorsalis (Hendel) (Enkerlin, this volume).

Barclay and van den Driessche (1989) also modelled this combination, and found that the two methods combine synergistically, especially when the fecundity and daily survivorship are both high. When the fecundity and survivorship are low, 
the synergism disappears. For parameter values approximating those of tsetse flies Glossina spp., synergism is reduced.

Knipling (1979) described the interaction of methyl eugenol (ME) for male annihilation used concurrently with the release of both sterile males and females, and found no interference and a high degree of synergism. Since the potential for development of resistance to ME has been demonstrated (Shelly 1997), it might also be possible to incorporate the use of sterile insects of the resistant strain, and thus increase effectiveness even more.

In the case where the attractant is non-sex-specific, such as with food baits, Barclay and van den Driessche (1989) showed that the two methods combine synergistically, especially when sterile insects are fed before release, and fecundity and survivorship are high. For parameter values approximating those of tsetse flies, there is still some synergism.

Barclay et al. (2014) also modelled the interaction of ME for male annihilation applied simultaneously with the release of ME-fed sterile males that are nonresponsive to ME male annihilation, using the same age-structured model as in section 2.12.2. They found that the two methods are synergistic. This is fortunate, as ME used alone appears not to be very useful in this context (Barclay and Hendrichs 2014), but elimination of wild males through male annihilation together with the SIT becomes very effective if the sterile males are raised with $\mathrm{ME}$ in their adult diet before release, after which they exhibit reduced attraction to ME traps and thus may come to dominate the male subpopulation over and above the numerical advantage they may achieve though high release rates. It appears that ME used alone can only be effective if trapping or response to male annihilation devices occurs prior to mating each day, which may or may not commonly occur in a control programme (Barclay and Hendrichs 2014). When ME is used simultaneously with the SIT, the order of mating and trapping is still important, but makes less difference than it does with ME alone.

Box 8. Combination of Sterile Releases and Pheromone Trapping
Miyai's model consisted of four differential equations: $d M / d t=F(a-b F)-c M-k M$;
$d V / d t=F(a-b F)-c V-\alpha[\min (M+S, V)] ; d F / d t=\alpha[\min (M+S, V)] M /(M+S)-c F$;
$d S / d t=R-c S-k S$; where $M, V, F$ and $S$ are the numbers of males, virgin females, fertilized females,
and sterile insects, respectively. The parameters are: $a$ is a density-independent fecundity, $b$ is a
density-dependent fecundity, $c$ is a death rate, $k$ is the rate of trapping of males, $\alpha$ is the mating
efficiency, and $R$ is the sterile insect release rate.

\subsubsection{SIT with Release of Parasitoids}

This combination has the advantage that parasitoids work well at high host densities, while the SIT works best at low pest densities. Knipling $(1979,1998)$ considered the release of both sterile males and females, and also Trichogramma sp., an egg parasitoid. His tables showed clearly that these two methods were synergistic. A recent field study found that the two methods in combination were more efficient than either method alone (Bloem et al. 1998). Especially in the case of inherited sterility, the release of partially sterile males and sterile females provides $F_{1}$ eggs and larvae for oviposition by egg and larval parasitoids, further augmenting the 
parasitoid populations (Marec et al., this volume). If both sterile insects and parasitoids are released inundatively (Marec et al., this volume), then each should become more efficient as the density declines, offering a powerful source of synergism (Knipling 1998).

Barclay (1987b) modelled the interaction of the inundative release of parasitoids and sterile insects using several variations on the usual host-parasitoid equations. This combination shows a high degree of synergism in all the models investigated, and appears to be close to ideal (Fig. 10). The main problems to be anticipated probably involve dispersal and phenology.

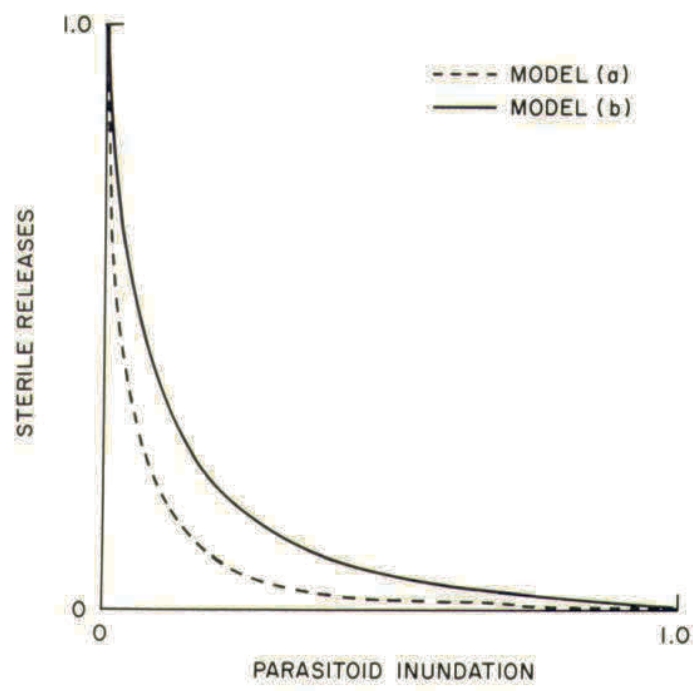

Figure 10. Critical release rates of sterile insects and parasitoids. All points on each curve are just sufficient to eradicate the pest population. Density-dependence is in the hosts in model (a), and in the parasitoids in model (b). (Figure from Barclay 1987c, reproduced with permission.)

\subsubsection{SIT with Pathogens}

The combination of the SIT and male-killing bacteria has been investigated by Berec et al. (2016) for bacteria that are transmitted vertically. They found that if vertical transmission is $100 \%$, then the SIT is not required. If transmission is less than $100 \%$, then the bacteria and the SIT act synergistically, and the combination is more efficient than either method alone. Specifically, with a relatively high rate of transmission of the bacteria, the required level of sterile male releases is much lower than without the bacteria. They investigated the interaction of bacteria with both non-heritable sterility (the usual SIT method employed for dipterans) and inherited sterility (used with lepidopterans) (Marec et al., this volume), and found that the efficiency of this combination for inherited sterility is best at intermediate doses of radiation. In New Zealand Suckling et al. (2007) integrated the use of Bt with sterile releases and achieved local eradication of the painted apple moth Teia anartoides 
Walker. Toledo et al. (2017) used Beauveria bassiana with an autoinoculation device for the control of Ceratitis capitata with a similar synergism.

\subsubsection{SIT with Sanitary Measures and Oviposition Traps}

If sanitation destroys oviposition habitat, and traps remove females that oviposit eggs, then there should be a complementary effect between the two, albeit moderated by density-dependence. If sterile males are also released, then the system has three sources of population reduction, none of which interferes with any other. Knipling (1979) calculated that these three should result in a highly efficient combination for control.

\subsubsection{Synergism between the SIT and a Natural Allee Effect}

The Allee effect (Allee 1931) is the phenomenon in which density-dependence becomes negative at very low population densities as a result of both sexes having difficulty in finding mates. It has since been generalized to include other sources of negative density-dependence as well (Yamanaka and Liebhold 2009). Although the Allee effect is not really a pest control method, it represents a natural weak point in the pest population dynamics (Suckling et al. 2012). Gordillo (2015a, b) has incorporated the Allee effect into models of the SIT, and noted the synergism that increasingly results with decreasing pest population, causing local extinctions.

Conversely, it may be possible for pockets of pest persistence to exist with sufficiently high dispersal even when the sterile release rate is above the predicted requirement for extinction, especially in heterogeneous habitats in which the number of sterile males depends on the estimated pest density. This is similar to the result of Levins and Culver (1971) in which populations could persist in a patchy environment in the face of local extinctions if dispersal is high enough, because some of the pest individuals disperse into neighbouring patches before extinction occurs in the home patch. Nevertheless, the dispersing pest population needs to be sufficiently high to overcome the Allee effect.

\subsection{Optimizing Initial Knockdown for the SIT}

Age at first oviposition is an important factor affecting spray intervals (Mangan and Bouyer, this volume). Adult insects usually take time to mature before mating and then ovipositing. If sprays targeting adults are timed so that the interval between sprays is smaller than the interval from adult emergence to first oviposition, then subsequently emerging adults will be killed before being allowed to oviposit. The number of sprays will depend on the time it takes from oviposition to adult emergence and number of generations per year or number of generations during the critical period required for population suppression. An example will illustrate this. In tsetse, the period between larviposition, pupation, and emergence of adults from the pupae is about 27 days at $27^{\circ} \mathrm{C}$, while the age to first larviposition is about 17 days following emergence of the adults. If the mean temperature is $27^{\circ} \mathrm{C}$, and the inter-spray interval is 15 days, and sprays kill $90 \%$ of the adult population, then the first spray will eliminate most of the adults, the second spray 15 days later will 
eliminate most of those adults that have emerged from the developing pupae, but before they start ovipositing, and a third spray will kill most of the adults that have emerged from the remaining pupae that were alive at the time of the first spray, again before they start ovipositing. The population is then in a suitable state to be controlled by sterile releases, as there will be little increase in the adult population for some time, long enough that the SIT should decrease the recruitment of juveniles and prevent this increase.

A model of tsetse developed by Barclay and Vreysen (2011) predicts the course of the adult population following two, three, and four applications of aerial insecticide sprays, each of which kills $98 \%$ of the adult population. For each of these three scenarios, a sterile release ratio (daily releases divided by the existing wild male population) of 0.2 was used, calculated immediately after the last spray. The tsetse example is presented because it is particularly clear. For most species, spray efficiency will be less than $100 \%$, and additional sprays will be required.

If sprays target larvae, then the length of the larval period is the factor that determines the time between sprays (Barclay et al. 2019). If sprays are 100\% efficient at killing the existing larvae, then a sufficient number of sprays to cover the entire life cycle will eliminate the population, if there is no immigration from outside the control area. If sprays are less than $100 \%$ efficient at killing larvae, which will usually be the case, then additional sprays will be required to bring the population down to a level suitable for the initiation of the SIT.

According to Barclay et al. (2019), an insecticide should be selected that will target the stage of longer length (larvae or adults), if that choice is available.

\subsection{Exclusion of Pests from an Area: Barrier Width}

Barclay et al. (2011) constructed a model that attempts to assess the minimum dimensions of a buffer area (within which control is also imposed) to protect a pest free area of low pest prevalence. Random dispersal would result in insects entering the buffer zone from outside the control area; these insects and their progeny would ultimately disperse across the buffer and enter the area to be protected unless they were killed within a sufficiently wide buffer. Dispersal in this case is modelled by means of diffusion equations, e.g. Okubo 1980, to model a pest population that is diffusing across the barrier and being controlled within the barrier in an effort to protect the inner core area inside. Their model considers a rectangular core area, surrounded by a rectangular buffer zone, and using the SIT as the control agent within the buffer. We are interested here mainly in determining the barrier width.

They assumed that (1) the core area is already a pest free area as a result of previous control efforts; (2) the host density in all areas (the core area, the buffer zone, and outside the buffer zone) is assumed to be at equilibrium; (3) there is a constant influx of pest insects from the region outside the buffer zone; and (4) no transport of the target pest insects by wind, storms, or humans into the core area occurs. As this treatment assumes that the equilibrium has already been achieved, the barrier width calculated is that required to maintain the status quo. Dispersal describes movement of the insects across the buffer zone and will determine the 
width of the buffer zone, which results in the density of the invading population approaching zero at the edge of the barrier zone adjacent to the core area.

\subsubsection{Description of the Diffusion Model}

The simplest 2-dimensional diffusion equation is the partial differential equation (Pielou 1969):

$$
\partial U(x, y, t) / \partial t=D\left(\partial^{2} U(x, y, t) / \partial x^{2}+\partial^{2} U(x, y, t) / \partial y^{2}\right)
$$

(see also Edelstein-Keshet 1988). This equation is a 2-dimensional analogue of an equation that was originally developed to describe the diffusion of heat along a metal rod (Fourier 1822), but has since been used for many other purposes as well, including insect dispersal. The diffusion coefficient, $D$, is defined in units of length squared per unit time, and is usually estimated by tabulating the linear difference between the initial and final positions resulting from insect dispersal, as well as the number of movements in a given time interval, and then computing the means of the squared net distances travelled per unit of time. For a population of insects released simultaneously at a point, equation (8) predicts an expanding Gaussian distribution with variance $4 D t$ at time $t$ :

$$
U(x, y, t)=(1 /(4 \pi D t))\left[\exp \left\{-\left(\left(x^{2}+y^{2}\right) / 4 D t\right)\right\}\right] \text { for } t>0
$$

Although most insect motion is demonstrably non-random, diffusion equations have been successful at predicting longer-term patterns of insect movement (Kareiva 1983; Turchin 1998) because population-level averaging occurs.

\subsubsection{Width of the Buffer Zone}

The pest population will have a certain density outside the buffer zone, and will disperse from outside into the buffer zone. If control measures are imposed within the buffer zone, the density of the pest will decrease from the outer edge of the buffer to the inner edge. The width of any buffer zone around a core area should be large enough to reduce the density of the pest close to zero in the core area. The buffer zone should be wide enough to prevent a gravid female insect and any of her offspring from crossing the buffer zone. Then an appropriate model is:

$$
\partial F(x, t) / \partial t=D\left(\partial^{2} F(x, t) / \partial x^{2}+\partial^{2} F(x, t) / \partial y^{2}\right)-g F(x, t)
$$

where $g$ is the growth function, $F$ is the female population density, $x$ is a spatial coordinate, and $t$ is time. If $g$ is linear, and births and deaths can be separated and are independent of $x$ and $t$, then:

$$
g F=\beta F-\delta F
$$


in which $\beta F$ and $\delta F$ are the instantaneous birth and death rates. Boundary conditions are such that at the outer edge of the buffer zone, $F(0, t)=F_{0}$, where $F_{0}$ is the density of insects at the outer edge of the buffer as a result of the influx of insects, and at the inside edge of the buffer, $F(w, t)=$ a small proportion of $F_{0}$ (e.g. $\left.10^{-6}\right)$, so that almost all the insects have died before reaching the inner side of the buffer. As a result of the continuous nature of the model, we can never actually achieve a zero density, but some small density below the Allee threshold that is non-viable will suffice.

If we are manipulating the death rate within the buffer by attract and kill devices that are evenly spread out to cover the whole of the buffer region, then $(\beta F-\delta F)$ will be negative, because now the second term in parenthesis consists of the sum of natural $(\delta)$ and imposed $(\mu)$ mortality from traps or other control sources. For control by killing, the kill rate $(\mu)$ may be combined multiplicatively with the natural mortality $(\delta)$ by multiplying survivorships (Barclay 1992b), so that if natural daily mortality is 0.05 and $10 \%$ of the population is killed each day, then $\delta+\mu$ becomes $1.0-(1.0-0.05)(1.0-0.10)=0.15-0.005=0.145$. The upper limit of total daily mortality is 1.0 and the net change is $\beta-1.0$, although this may be impossible to achieve or even approach.

If the SIT is used as the control method, then we are manipulating $\beta$, and it will decrease from its natural value as sterile male releases increase. In this case, $(\beta F$ $\delta F)$ is also negative if control is to be effective. To simplify the solution of the diffusion equation when using the SIT, it will be assumed that the release of sterile males is proportional to the size of the wild insect population at all points within the buffer. The dynamics of this situation has been described by Knipling (1960), Hawkes and Coaker (1977), and Barclay (1981a), among others. Using this simplification, the same development described below will be useful for both killing insects or reducing reproduction. For control by SIT integration, the natural fertility is multiplied by a factor equal to the fertility factor, $\sigma=F /(M+S)$ in the SIT equation. If the sex ratio is $1: 1$, then the fertility factor is $M /(M+S)$. Thus, if $M=$ 100 and $S=200$, and if natural daily fertility is 0.10 , then $\beta \sigma$ becomes $0.10[100 /$ $(100+200)]=0.033$. The lower limit of suppression here occurs as $\sigma \rightarrow 0.0$, so the net change is $0.0-\delta$, although this also may be impossible. Even with maximal SIT action, the rate of change of population density with distance across the buffer is likely to be slow.

Since we assume that we are dealing with an equilibrium situation, in which the insects have been diffusing and the buffer has been under control for a long time, the time derivative is zero, as nothing is changing over time. Only the space derivative is still non-zero. This yields the equilibrium equation:

$$
d F / d x=(\delta-\beta \sigma) F
$$

and this has solutions proportional to $e^{-\varphi x}$, where $\varphi^{2}=(\delta-\beta \sigma) / D$. Assuming the insect density gradient across the buffer is $F(x)=c e^{-\varphi x}$, the boundary conditions dictate that $c=F_{0}$ and $F_{0} e^{-\varphi w}=10^{-6} F_{0}$. Taking logarithms, $-\varphi w=\ln \left(10^{-6}\right)=-13.8$. This leads to the minimum buffer width $(w)$ :

$$
w=13.8 / \varphi=13.8 /[(\delta-\beta \sigma) / D]^{1 / 2}
$$


to reduce the population at the inner edge of the buffer to one millionth of that at the outer edge. If a decrease down to $10^{-6}$ of the original density outside the buffer $\left(F_{0}\right)$ is not satisfactory, then some other small fraction can be chosen, e.g. for $10^{-5}$ the 13.8 becomes 11.5 , and for $10^{-7}$ the 13.8 becomes 16.1 . The units of $w$ in eq. (13) are in the units of $D$, and the units of $\beta$ and $\delta$ must be the same as those of $D$.

For the case of killing insects, (13) becomes:

$$
w=13.8 /[(\delta+\mu)-\delta \mu-\beta) / D]^{1 / 2}
$$

It is clear that, for control by SIT integration, the value of $\sigma$ must be less than $\delta / \beta$ to cause a reduction in the population. Thus, $\sigma<\delta / \beta$ implies that $M /(M+S)<\delta / \beta$, and this implies that $S>M(\delta / \beta-1)$. Graphs of $w$ vs. $\mu$ and $w$ vs. $\sigma$ are shown in Fig. 11 for two values of the diffusion coefficient, two values of $\delta-\beta$, and one value of acceptable density on the inner edge of the buffer, $10^{-6}$.
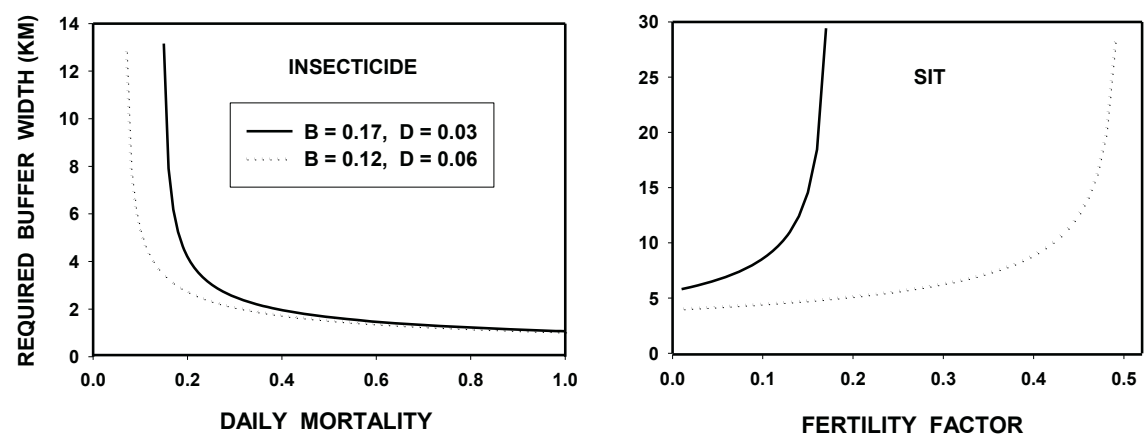

Figure 11. Estimates of required buffer widths to reduce the population density at the inner edge of the buffer to $10^{-6}$ of that at the outer edge. The curves show buffer width as a function of daily mortality (left panel) or fertility factor using SIT application (right panel) for daily death and birth rates that might be appropriate to the Mediterranean fruit fly. The legend values apply to both panels.

Control by the SIT and by insecticidal traps are not commensurate, as they use different mechanisms, but they can be compared by forming an equivalence in which the value of $w$ using the SIT equals the value of $w$ using traps. Equating (13) and (14) we obtain: $\left.13.8 /[(\delta-\beta \sigma) / D]^{1 / 2}=13.8 /[(\delta+\mu)-\delta \mu-\beta) / D\right]^{1 / 2}$, which simplifies to

$$
\sigma=[\beta-\mu(1-\delta)] / \beta
$$

The parameter $\sigma$ is the fertility factor, $M /(M+S)$, so that to achieve a buffer width equal to that using a daily trapping rate of $\mu$, an overflooding ratio of $\mathrm{S} / \mathrm{M}=$ $(1-\sigma) / \sigma$ needs to be maintained. Note that, in a continuously reproducing population, the overflooding ratio is not necessarily the release ratio (section 2.12.3.). The release ratio (number of sterile males released each time period divided 
by the total number of wild males present) is related to the overflooding ratio as follows. If the daily mortality of the wild males is $q$ and that of sterile males is $r$, and if the daily recruitment rate of wild males is $m$, and the daily release rate of sterile males is $n$, and if the survivorship curves of both are exponential (geometric if measured by days), and if the lifetimes of both are unlimited, then the total number of wild males in the population at equilibrium is obtained from an infinite geometric series, $M=m /(1-q)$, and the total number of sterile males is $S=n /(1-n)$, so that the overflooding ratio is $[n /(1-r)] /[m /(1-q)]=(n / m)[(1-q) /(1-r)]$. If the daily survivorships of wild and sterile males are equal, then this reduces to an overflooding ratio of $n / m$, i.e. the quotient of the recruitments, which will also be the quotient of the wild and sterile male population sizes at equilibrium.

If neither traps nor the SIT yield reasonable buffer widths, it is possible to use both traps and the SIT in combination and further reduce the buffer width. It is true that the traps are likely to attract sterile males as well as fertile males, but if sterile and fertile males are equally attracted to the traps, the overflooding ratio will not change, but the wild population will be reduced by both control methods. In that case, the buffer width is:

$$
w=13.8 /[(\delta+\mu)-\delta \mu-\beta \sigma) / D]^{1 / 2}
$$

and this is finite and positive only if $\delta+\mu-\delta \mu>\beta \sigma$, i.e. if mortality is greater than fertility.

Gordillo (2014) extended this approach to include optimization of sterile releases on the basis of the cost of sterile males for a density-dependent population, and including the possibility of variable sterile-male release rates. He used a onedimensional diffusion equation and a numerical process called "simulated annealing", and concluded that in some cases sterile release rates should be uniformly high near the high-pest-density boundary of the buffer, and then drop sharply to much lower levels towards the core area to be protected.

\subsubsection{Case Study: Mediterranean Fruit Fly}

Carey (1989) gave the following daily values for C. capitata: $\beta=0.17$ and $\delta=0.03$, and $r=0.14$. The diffusion coefficient, $D$, has been estimated by Plant and Cunningham (1991) to be $D=0.006 \mathrm{~km}^{2} /$ day. It is clear that, if the kill rate or reduction in fertility is less than 0.14 per day, then the number of new daily recruits is still greater than the natural death rate. If the daily kill rate, $\mu$, is 0.20 , then using eq. (14), the minimum buffer width is $w=13.8 / \sqrt{ }[(0.23-0.17) / 0.006)]=4.36$ $\mathrm{km}$.

If the SIT is the control method, and if the overflooding ratio is $10: 1$, then $\sigma=$ 0.091 , so the minimum buffer width is $w=13.8 / \sqrt{ }[(0.03-0.17(0.09)) / 0.006]=$ $8.87 \mathrm{~km}$. If both insecticidal traps and the SIT are used, then the buffer width is: $w=$ $13.8 / \sqrt{ }[(0.23-0.17(0.091)) / 0.006]=2.31 \mathrm{~km}$. This compares with the use of traps only, at twice the kill rate $(0.40)$, which yields a buffer width of $2.10 \mathrm{~km}$, or the use of the SIT at twice the overflooding ratio $(\sigma=0.045)$, which yields a buffer width of $7.29 \mathrm{~km}$. Since it appears unlikely that traps can catch $40 \%$ of the insects daily, the combination of the SIT and trapping appears potentially to be useful. 
Recent studies of Mediterranean fruit fly dispersal suggest that a 2-km buffer zone is a reasonable first guess of the buffer width. Meats and Smallridge (2007) found that $90 \%$ of released flies remained within $0.4-0.7 \mathrm{~km}$ of the release point. It has been shown in Guatemala that most adult flies disperse short distances of only a few hundred metres, although some can disperse on winds up to $51 \mathrm{~km}$ from the release point (Villatoro 2014; Enkerlin et al. 2016), and it may be possible for flies to spread considerable distances when the food resource is depleted. Thus, a 2-km buffer might be useful if the aim of control was simply to yield a low-level pest infestation in a low-level horticultural production area. However, a much wider buffer will likely be more realistic, especially if the aim is complete exclusion of the pest from a protected area. In addition, the diffusion model used here assumes random movement, and many insects move quite non-randomly under conditions of stress or lack of food. We stress that the parameter values we have used are used for illustrative purposes only; any real control programme should use data obtained from the control area as well as any information on long-distance movement.

For the Mediterranean fruit fly example above, it would seem that control must be quite high to achieve a reasonable buffer width. This implies that a combination of synergistic controls would probably be advisable. A mitigating factor in these estimates of barrier width is that the formulas were developed assuming that the overflooding ratio would be constant across the barrier, rather than the sterile release rate being constant as the wild population decreases toward the inner edge of the barrier. This assumption would have the effect of increasing the estimate of the required barrier width. As the model appears to be intractable with a constant sterile release rate across the barrier width, a more reasonable approach might be to calculate $S$ using the population density at the uncontrolled edge of the barrier and to use that value throughout the barrier; this would reduce the required barrier width.

\subsection{Optimization of Programme Releasing Sterile Insects}

Optimization inevitably involves economics (Mumford, this volume). Although that is somewhat outside the scope of this chapter, a beginning has been made on this topic. Geier (1969) used demographic models incorporating density-dependence to analyse the efficiency of programmes that release sterile insects, and to derive optimal strategies for control. Barclay and Li (1991) used a general treatment of combinations of pest control to determine optimal proportions of each control method. Atzeni et al. (1992) examined the situation for the Old World screwworm Chrysomya bezzania (Villeneuve), and included buffer width, male competitiveness, and population aggregation in their analysis. Anaman et al. (1994) performed a benefit/cost analysis with $C$. bezzania, and incorporated beef losses into the equation. Gordillo (2014) addressed the optimization question for sterile releases in a buffer zone that protects an insect-free area, and Ramirez and Gordillo (2016) used benefit/cost analysis to optimize sterile releases for insects that are long-lived but have short reproductive periods, such as some beetles. 


\subsection{Development of Resistance}

There has been a marked tendency for insects to develop resistance to insecticides or other control methods. It is conceivable that a wild pest population could develop resistance to the use of sterile releases as a means of control (Itô et al., this volume; Lance and McInnis, this volume; Hendrichs and Robinson, this volume; Whitten and Mahon, this volume). This resistance might involve behavioural mechanisms that would preclude the sterile-fertile matings (Barclay 1990). Selection for resistance to several pest control methods operating together has been modelled by Barclay (1996), and it appears that selection for resistance to a particular control method is a linear function of the amount of mortality being inflicted by that control method, and the degree of isolation of the targeted population.

\section{PARAMETER ESTIMATION FOR THE MODELS}

Knowledge of several parameters is crucial to the success of any programme that releases sterile insects. With reference to the models outlined above, the basic parameters that will always be of interest are: $F$, the population size; $\lambda$, the potential rate of population increase each generation; $q_{m}$ and $q_{f}$, the proportions of the released males and females that remain fertile; and $c$, the competitive ability of sterile males relative to the wild fertile male population. Some of the estimations can be done using standard population biology methods. The population size can be crudely estimated from trap catches. Hargrove (1990) used mark-recapture techniques to estimate the size of tsetse fly populations (Weidhaas 1973; Itô et al., this volume). The rate of increase, $\lambda$, would normally be determined using oviposition rates. The residual fertilities, $q_{m}$ and $q_{f}$, could be determined by caging sterile males with fertile females, and fertile males with sterile females, either in groups or pairs, and noting the resulting fertile egg production. Competitive ability of sterile males, $c$, could then be determined from the laboratory, but preferably from field cage or small-scale field experiments, where immigration could be assumed to be negligible, using the equation involving competitive ability, and then solving for $c$. The information on $\lambda$, $q_{m}$ and $q_{f}$ must be determined first, or the equation becomes confounded. Alternatively, Meats (1998) used release and recapture techniques to estimate the quality of released sterile insects. Immigration into the control area could then be determined using either mark-recapture or the equation involving immigration and solving for $v$. Plant and Cunningham (1991) detailed procedures for estimating the dispersal of Mediterranean fruit flies, and estimates of immigration could be obtained from considerations of dispersal.

The determination of density-dependence is problematic; there are many models of density-dependence, and none of them is particularly mechanistic. Thus rates of oviposition and subsequent survivorship would have to be monitored at various densities to derive a function to describe the depressing effects at various levels. In many wild populations, even just detecting the existence of density-dependence is difficult, much less the quantification of depressing effects. However, in view of the potential assistance to the SIT, an estimation of the effects of density-dependence is 
worthwhile. Itô et al. (this volume) develop further the subject of parameter estimation.

\section{ASSESSMENT OF SIT MODELLING}

\subsection{Uses of Models}

Models can be used to predict and explain the behaviour of a population. This information guides research, generates hypotheses, and aids teaching. Most models of the SIT have so far have been aimed mainly at predicting the behaviour of pest populations under various constraints, such as incomplete sterility, lack of competitive ability of sterile males, the immigration of wild insects into a control area, etc. One of the best uses of models is to generate ideas or hypotheses that are capable of experimental testing. Thus, ideally, modelling should go hand-in-hand with field and laboratory experiments to verify or falsify a model's predictions.

There have been a number of computer simulations of the SIT for the purpose of instruction in both the technique and in insect population dynamics generally. At least two have been documented and are available to the public. Both represent various elaborations on the original model of Knipling.

Sawyer et al. (1987) described a simulation that includes spatial heterogeneity, aggregation, immigration, random effects, and reduced sterile male competitiveness. This model was adapted by Flanders and Arneson (2018) under the name "Curaçao", and is available for downloading. The user can specify the various parameters and options, and then compare runs to draw conclusions.

Potgieter et al. $(2013,2016)$ aimed their models at a particular lepidopteran insect, and incorporated some of the features described above. Such models are valuable in any SIT programme against a particular insect.

\subsection{Advantages and Limitations of Modelling}

The models of the SIT constructed thus far fall generally into three groups: (1) models that investigate processes that determine the proportion of eggs laid that are sterile, (2) models involving population dynamics and other population level phenomena, and (3) models that investigate the interactions of the SIT with other control methods, although it might be argued that the last two really belong together. The first category, including residual sterility, reduced sterile competitive ability, mating patterns, and immigration, is of crucial importance in planning and executing a programme that releases sterile insects. Unless one can accurately predict the level of sterility in eggs produced by the wild females, the programme is liable to fail. In addition, it is here that models are most likely to give realistic answers, as these processes rely mostly on determinable proportions or coefficients, rather than somewhat nebulous population processes.

Models of more general aspects of population dynamics involve many hidden factors, such as the strength of density-dependence, the functional responses of predators, synchrony of pest and predator phenologies, the degree of pest population 
aggregation, the extent to which sterile insects assume the same spatial patterns as the wild insects, etc. These are not easy to determine, and the models in the second and third categories must be taken as heuristic, rather than quantitatively predictive. They provide insights into the kinds of responses to expect, but quantitative accuracy must await species-specific simulations based on accurate and detailed biological and ecological information regarding the whole system.

\subsection{Transient versus Equilibrium Models}

Many analytic models of the SIT are solved for equilibrium, and the results of the parameters on the equilibrium are noted. In real life, populations are almost always changing. Analysis of the equilibrium behaviour certainly has much to say about the effects of the parameters on the transient behaviour as well as on the equilibrium, but a proper analysis should include the effects of the parameters on the dynamics of transient behaviour. The problem is that there is an infinite number of trajectories that any population can follow, and to encapsulate the behaviour of these in digestible form is no small task. One criterion that can be used is that of stability of the system, in its various forms. Stability characteristics can be related to parameter ranges, and certain characteristics of the resulting transient behaviour can be inferred from them.

\subsection{Future Directions and Information Needs}

The models reviewed in this chapter cover most of the relevant topics in the dynamics of the SIT. However, it is only a good beginning, and there is much left to do. Models that have a more realistic ecological basis will be required to suggest new hypotheses and to give more accurate predictions of behaviour. One area still largely untapped is metapopulation models - models including patches with migration among patches, local extinctions and re-establishments. A start has been made with the models including immigration, heterogeneity, and diffusion (Lewis and van den Driessche 1993; Gordillo 2015a). The next step is to tie these together into a meaningful whole.

Another area that will yield useful information is the construction of speciesspecific models for the SIT, including all relevant factors. Many species-specific models have been constructed, but many appear to have inadequate detailed ecological information. In addition, the area of behavioural ecology will probably emerge as being especially relevant.

Testing models, experimentally and in the field, is in its infancy. Information is needed on the effects of pest density-dependent regulation on the efficiency of the SIT, the effects of predators and parasitoids on the dynamics of the SIT, and the effects of ecosystem resilience. The simultaneous use of other control methods with the SIT is still largely hypothetical, and this potentially useful area needs considerable investigation. 


\section{REFERENCES}

Ailam, G., and R. Galun. 1967. Optimal sex ratio for the control of insects by the sterility method. Annals of the Entomological Society of America 60: 41-43.

Allee, W. C. 1931. Animal aggregation. University of Chicago Press, Chicago, IL, USA.

Anaman, K. A., M. G. Atzeni, D. G. Mayer, and M. A. Stuart. 1994. Benefit-cost analysis of the use of sterile insect technique to eradicate screwworm fly in the event of an invasion from Australia. Preventive Veterinary Medicine 20: 79-98.

Atzeni, M. G., D. G. Mayer, and D. G. Butler. 1992. Sterile insect release method - optimal strategies for eradication of screwworm fly. Mathematics and Computers in Simulation 33: 445-450.

Bakhoum, M. T., M. J. B. Vreysen, and J. Bouyer. 2021. The use of species distribution modelling and landscape genetics for tsetse control, pp. 857-868. In J. Hendrichs, R. Pereira and M. J. B. Vreysen (eds.), Area-wide integrated pest management. Development and field application. CRC Press, Boca Raton, FL, USA.

Barclay, H. J. 1980a. The sterile insect release method for species with two-stage life cycles. Researches on Population Ecology 21: 165-180.

Barclay, H. J. 1980b. Models for the sterile insect release method with the concurrent release of pesticides. Ecological Modelling 11: 167-178.

Barclay, H. J. 1981a. Population models on the release of chemosterilants for pest control. Journal of Applied Ecology 18: 679-695.

Barclay, H. J. 1981b. The sterile insect release method for a pest species in competition with another species. Researches on Population Ecology 23: 145-155.

Barclay, H. J. 1982a. The sterile release method with unequal male competitive ability. Ecological Modelling 15: 251-263.

Barclay, H. J. 1982b. Population stability under sterile releases. Researches on Population Ecology 24: 405-416.

Barclay, H. J. 1984. Demographic consequences of monogamy and polygamy for a sterile release programme. Protection Ecology 6: 209-217.

Barclay, H. J. 1987a. Models of sterile insect releases for populations under attack by parasitoids. Ecological Modelling 36: 155-169

Barclay, H. J. 1987b. Models for pest control: complementary effects of periodic releases of sterile pests and parasitoids. Theoretical Population Biology 32: 76-89.

Barclay, H. J. 1987c. Combining methods of pest control: complementarity of methods and a guiding principle. Natural Resource Modeling 2: 299-323.

Barclay, H. J. 1990. Uncertainties associated with release agents for autocidal control, pp. 185-193. In J. Marois and R. Plant (eds.), Risk assessment in agricultural biotechnology. University of California, Davis, CA, USA.

Barclay, H. J. 1992a. Modelling the effects of aggregation on the efficiency of insect pest control. Researches on Population Ecology 34: 131-141.

Barclay, H. J. 1992b. Combining methods of insect pest control: partitioning mortality and predicting complementarity. Researches on Population Ecology 34: 91-107.

Barclay, H. J. 1996. Combining methods of insect pest control: modelling selection for resistance to control methods in combination. Researches on Population Ecology 38: 75-85.

Barclay, H. J. 2001. Modeling incomplete sterility in a sterile release program: interactions with other factors. Researches on Population Ecology 43: 197-206.

Barclay, H. J. 2016. Determination of the sterile release rate for stopping growing age-structured populations. International Journal of Pest Management 62: 40-54. http://dx.doi.org/10.1080/09670874.2015.1115913

Barclay, H., and C. Li. 1991. Combining methods of pest control: minimizing cost during the control programme. Theoretical Population Biology 40: 105-123.

Barclay, H., and M. Mackauer. 1980a. The sterile insect release method for pest control: a densitydependent model. Environmental Entomology 9: 810-817.

Barclay, H., and M. Mackauer. 1980b. The sterile insect release method for species under predation or parasitism. Researches on Population Ecology 22: 136-146.

Barclay, H. J., and P. van den Driessche. 1989. Pest control models of combinations of sterile releases and trapping. Insect Science and its Application 10: 107-116.

Barclay, H. J. and P. van den Driessche. 1990. A sterile release model for control of a pest with two life stages under predation. Rocky Mountain Journal of Mathematics 20: 847-855. 
Barclay, H. J., and M. J. B. Vreysen. 2011. A dynamic population model for tsetse (Diptera: Glossinidae) area-wide integrated pest management. Population Ecology 53: 89-110. https://link.springer.com/article/10.1007/s10144-010-0224-7?no-access=true

Barclay, H. J., and M. J. B. Vreysen. 2013. The interaction of dispersal and control methods for the riverine tsetse fly Glossina palpalis gambiensis (Diptera: Glossinidae): a modelling study. Population Ecology 55: 53-68. https://link.springer.com/article/10.1007/s10144-012-0339-0?no-access=true

Barclay, H. J., and J. Hendrichs. 2014. Models for assessing the male annihilation of Bactrocera spp. with methyl eugenol baits. Annals of the Entomological Society of America 107: 81-96. https://doi.org/10.1603/AN13046

Barclay, H. J., R. Matlock, S. Gilchrist, D. M. Suckling, J. Reyes, W. R. Enkerlin, and M. J. B. Vreysen. 2011. A conceptual model for assessing the minimum size area for an area-wide integrated pest management program. International Journal of Agronomy 2011, Article ID 409328, 12 pp. http://dx.doi.org/10.1155/2011/409328 https://www.hindawi.com/journals/ija/2011/409328/

Barclay, H. J., D. McInnis, and J. Hendrichs. 2014. Modeling the area-wide integration of male annihilation and the simultaneous release of methyl eugenol-exposed Bactrocera spp. sterile males. Annals of the Entomological Society of America 107: 97-112. https://doi.org/10.1603/AN13010

Barclay, H. J., R. Steacy, W. Enkerlin, and P. van den Driessche. 2016. Modeling diffusive movement of sterile insects released along aerial flight lines. International Journal of Pest Management 62: 228-244. http://dx.doi.org/10.1080/09670874.2016.1174319

Barclay, H. J., J. W. Hargrove, R. Steacy, and P. van den Driessche. 2019. Modelling optimal timing and frequency of insecticide sprays for knockdown in preparation for other control measures. International Journal of Pest Management 65(2): 114-129. https://doi.org/10.1080/09670874.2018.1475690

Barclay, H. J., J. W. Hargrove, and P. van den Driessche. 2020. Estimating tsetse fertility: daily averaging versus periodic larviposition. Medical and Veterinary Entomology 34: 27-33. https://doi.org/10.1111/mve.12399

Berec, L., D. Maxin, and V. Bernhauerova. 2016. Male-killing bacteria as agents of insect pest control. Journal of Applied Ecology 53: 1270-1279. http://onlinelibrary.wiley.com/doi/10.1111/1365-2664.12638/abstract

Berryman, A. 1967. Mathematical description of the sterile male principle. The Canadian Entomologist 99: $858-865$.

Berryman, A. A., T. P. Bogyo, and L. C. Dickmann. 1973. Computer simulation of population reduction by release of sterile insects: II. The effects of dynamic survival and multiple mating, pp. 31-43. In Proceedings, Panel: Computer Models and Application of the Sterile-Male Technique. Joint FAO/IAEA Division of Atomic Energy in Food and Agriculture, 13-17 December 1971, Vienna, Austria. STI/PUB/340. IAEA, Vienna, Austria. https://www.iaea.org/publications/3096/computer-models-and-application-of-the-sterile-maletechnique-vienna-13-17-dec-1971

Bloem, S., K. A. Bloem, and A. L. Knight. 1998. Oviposition by sterile codling moths, Cydia pomonella (Lepidoptera: Tortricidae) and control of wild populations with combined releases of sterile moths and egg parasitoids. Journal of the Entomological Society of British Columbia 95: 99109. https://journal.entsocbc.ca/index.php/journal/article/view/479

Bogyo, T. P. 1975. Mathematical models and computer simulation of insect control, pp. 161-168. In Proceedings, Symposium: Sterility Principle for Insect Control 1974. International Atomic Energy Agency and Food and Agriculture Organization of the United Nations, 22-26 July 1974, Innsbruck, Austria. STI/PUB/377. IAEA, Vienna, Austria. https://www.iaea.org/publications/3142/sterility-principle-for-insect-control-1974

Bogyo, T. P., A. A. Berryman, and T. A. Sweeney. 1971. Computer simulation of population reduction by release of sterile insects. I. A study of the relative importance of the parameters of a mathematical model, pp. 19-25. In Proceedings, Panel: Application of Induced Sterility for Control of Lepidopterous Populations. Joint FAO/IAEA Division of Atomic Energy in Food and Agriculture, 1-5 June 1970, Vienna, Austria. STI/PUB/281. IAEA, Vienna, Austria.

Carey, J. R. 1989. Demographic analysis of fruit flies, pp. 253-265. In A. S. Robinson and G. Hooper (eds.), World crop pests, volume 3B. Fruit flies. Their biology, natural enemies and control. Elsevier, Amsterdam, The Netherlands.

Carpenter, J. E. 2013. Integrating augmentative biological control and inherited sterility for management of lepidopteran pests, pp. 101-103. In P. G. Mason, D. R. Gillespie and C. Vincent (eds.), Proceedings of the $4^{\text {th }}$ International Symposium on Biological Control of Arthropods, 4-8 
March 2013, Pucón, Chile. 381 p. https:/www.cbd.int/invasive/doc/meetings/isaem-201501/BIOCONTROL/Expert\%20Submissions/Sheppard/iasem-expert-sheppard-bio-08-en.pdf

Costello, W. G., and H. M. Taylor. 1975. Mathematical models of the sterile male technique of insect control, pp 318-359. In A. Charnes and W. R. Lynn (eds.), Mathematical analysis of decision problems in ecology. Lecture Notes in Biomathematics, Volume 5. Springer-Verlag, Berlin, Germany.

Dietz, K. 1976. The effect of immigration on genetic control. Theoretical Population Biology 9: 58-67.

Edelstein-Keshet, L. 1988. Mathematical models in biology. Random House, New York, USA.

Enkerlin, W., A. Villaseñor, S. Flores, D. Midgarden, E. Lira, P. Rendon, J. Hurley, E. Salazar, W. Méndez, R. Castañeda, E. Cotoc, J. L. Zavala, H. Celedonio, and J. M. Gutiérrez Ruelas. 2016. Descriptive analysis of the factors affecting population fluctuation of the Mediterranean fruit fly (Ceratitis capitata, Wied.) in coffee areas located in Guatemala and its implications in IPM strategies, pp. 46-63. In B. Sabater-Muñoz, T. Vera, R. Pereira, and W. Orankanok (eds.), Proceedings of the 9th International Symposium on Fruit Flies of Economic Importance, 12-16 May 2014, Bangkok, Thailand. ISBN 978-616-358-207-2.

https://nucleus.iaea.org/sites/naipc/twd/Documents/Proceedings_9thISFFEI.pdf

(FAO/IAEA) Food and Agriculture Organization of the United Nations/International Atomic Energy Agency. 2016. Guidelines for the use of mathematics in operational area-wide integrated pest management programmes using the sterile insect technique with a special focus on tephritid fruit flies. H. J. Barclay, W. Enkerlin H., N. C. Manoukis and J. Reyes Flores (eds.), Food and Agriculture Organization of the United Nations, Rome, Italy. $95 \mathrm{pp}$. http://www-naweb.iaea.org/nafa/ipc/public/tephritid-fruit-flies-manual.pdf

Flanders, K. L., and P. A. Arneson. 2018. The sterile insect release method - a simulation exercise. Curaçao. In Radcliff's IPM world textbook. University of Minnesota, St. Paul, MN, USA. https://ipmworld.umn.edu/flanders

Flores, J. C. 2003. A mathematical model for wild and sterile species in competition: immigration. Physica A: Statistical Mechanics and its Application 328: 214-224. https://doi.org/10.1016/S0378-4371(03)00545-4

Fourier, J. 1822. Analytical theory of heat. Dover, New York, USA.

Geier, P. W. 1969. Demographic models of population response to sterile-release procedures for pest control, pp 33-41. In Proceedings, Panel: Insect Ecology and the Sterile-Male Technique. Joint FAO/IAEA Division of Atomic Energy in Food and Agriculture, 7-11 August 1967, Vienna, Austria. STI/PUB/223. IAEA, Vienna, Austria. https:/www.iaea.org/publications/2197/insect-ecology-and-the-sterile-male-technique

Gordillo, L. F. 2014. Optimal sterile insect release for area-wide integrated pest management in a density regulated pest population. Mathematical Biosciences and Engineering 11: 511-521. DOI: 10.3934/mbe.2014.11.511 www.aimsciences.org/journals/displayArticlesnew.jsp?paperID=9554

Gordillo, L. F. 2015a. Pest persistence and eradication conditions in a deterministic model for sterile insect release. Journal of Biological Dynamics 9(Suppl. 1): 64-78. http://dx.doi.org/10.1080/17513758.2014.942393

Gordillo, L. F. 2015b. Modeling ephemeral mating encounters in insects: the emergence of matefinding Allee effects and applications to theoretical models of sterile release. Theoretical Population Biology 104: 10-16. https://doi.org/10.1016/j.tpb.2015.06.002

Hamada, R., and S. Miyai. 1985. Theory of eradication, pp 79-97. In S. Ishi, K. Kiritani and T. Kocha (eds.), Eradication of fruit flies: theory and practice. (In Japanese). Japan Agriculture Aviation Association, Tokyo, Japan.

Hargrove, J. W. 1990. Population estimation from mark-recapture data. Equations for a pooled mark system and for pooled data, with applications to a study on island populations of tsetse flies in Zimbabwe, pp. 79-114. In Proceedings, Panel: Sterile Insect Technique for Tsetse Control and Eradication. Final Research Co-ordination Meeting, Joint FAO/IAEA Division of Nuclear Techniques in Food and Agriculture, 6-10 June 1988, Vom, Plateau State, Nigeria. STI/PUB/830. IAEA, Vienna, Austria. https://www.iaea.org/publications/3698/sterile-insect-technique-for-tsetse-control-and-eradication

Harrison, G. W., H. J. Barclay, and P. van den Driessche. 1982. Analysis of an insect release model with predation. Journal of Mathematical Biology 16: 33-47.

Hassell, M. P. 1978. The dynamics of arthropod predator-prey systems. Princeton University Press, Princeton, NJ, USA. 
Hawkes, C., and T. H. Coaker. 1977. A model for chemosterilization experiments with special reference to the cabbage root fly (Erioischia brassicae (Bouche)). Journal of Applied Ecology 14: 43-53.

Hendrichs, J., B. I. Katsoyannos, and V. Wornoayporn. 1994. Odour-mediated foraging by yellowjacket wasps (Hymenoptera: Vespidae): predation on leks of pheromone-calling Mediterranean fruit fly males (Diptera: Tephritidae). Oecologia 99: 88-94.

https://link.springer.com/article/10.1007/BF00317087?no-access=true

Hendrichs, J., G. Franz, and P. Rendon. 1995. Increased effectiveness and applicability of the sterile insect technique through male-only releases for control of Mediterranean fruit flies during fruiting seasons. Journal of Applied Entomology 119: 371-377. http://onlinelibrary.wiley.com/doi/10.1111/j.14390418.1995.tb01303.x/abstract;jsessionid=FDF26C5E844878A695304112AECD43C8.f02t02

Hendrichs, J., A. S. Robinson, J. P. Cayol, and W. Enkerlin. 2002. Medfly areawide sterile insect technique programmes for prevention, suppression or eradication: the importance of mating behavior studies. Florida Entomologist 85: 1-13. http://journals.fcla.edu/flaent/article/view/75040/72698

Hendrichs, M. A., and J. Hendrichs. 1998. Perfumed to be killed: interception of Mediterranean fruit fly (Diptera: Tephritidae) sexual signaling by predatory foraging wasps (Hymenoptera: Vespidae). Annals of the Entomological Society of America 91: 228-234.

DOI: 10.1093/aesa/91.2.228 https:/www.researchgate.net/publication/233657949

Hendrichs, M. A., V. Wornoayporn, B. Katsoyannos, and J. Hendrichs. 2007. Quality control method to measure predator evasion in wild and mass-reared Mediterranean fruit flies (Diptera: Tephritidae). Florida Entomologist 90: 64-70. http://www.jstor.org/stable/4151139

Horng, S. B., and R. E. Plant. 1992. Impact of lek mating on the sterile insect technique: a modeling study. Researches on Population Ecology 34: 57-76.

Itô, Y. 1977. A model of sterile insect release for eradication of the melon fly, Dacus cucurbitae Coquillett. Applied Entomology and Zoology 12: 303-312.

Itô, Y., and H. Kawamoto. 1979. Number of generations necessary to attain eradication of an insect pest with the sterile insect release method: a model study. Researches on Population Ecology 20: 216-226.

Iwahashi, O. 1977. Eradication of the melon fly, Dacus cucurbitae, from Kume Is., Okinawa with the sterile insect release method. Researches on Population Ecology 19: 87-98.

Kareiva, P. M. 1983. Local movement in herbivorous insects: applying a passive diffusion model to mark-recapture field experiments. Oecologia 57: 322-327. http://www.jstor.org/stable/4216967

Kean, J. M., A. E. A. Stephens, S. L. Wee, and D. M. Suckling. 2007. Optimizing strategies for eradication of discrete-generation lepidopteran pests using inherited sterility, pp. 211-220. In M. J. B. Vreysen, A. S. Robinson and J. Hendrichs (eds.), Area-wide control of insect pests. From research to field implementation. Springer, Dordrecht, The Netherlands. http://www-naweb.iaea.org/nafa/ipc/public/Area-Wide-Control-Insect-Pests-book.pdf

Kimanani, E. K., and J. W. Odhiambo. 1993. A stochastic model for the sterile male technique. Biometrics 49: 577-585.

Klassen, W., and J. F. Creech. 1971. Suppression of pest population with sterile male insects. Miscellaneous Publication, Number 1182. USDA/ARS, Washington, DC, USA.

Klassen, W., J. V. Adams, and M. B. Twohey. 2004. Modeling the suppression of sea lamprey populations by the release of sterile males or sterile females. Journal of Great Lakes Research 30: $463-473$.

Knipling, E. F. 1955. Possibilities of insect control or eradication through the use of sexually sterile males. Journal of Economic Entomology 48: 459-462.

Knipling, E. F. 1959. Sterile male method of population control. Science 130: 902-904.

Knipling, E. F. 1960. Use of insects for their own destruction. Journal of Economic Entomology 53: 415-420.

Knipling, E. F. 1964. The potential role of the sterility method for insect population control with special reference to combining this method with conventional methods. USDA/ARS (Series) 33-98.

Knipling, E F. 1979. The basic principles of insect population suppression and management. Agriculture Handbook Number 512. USDA/SEA, Washington, DC, USA.

Knipling, E. F. 1998. Role of parasitoid augmentation and sterile insect techniques in areawide management of agricultural insect pests. Journal of Agricultural Entomology 15: 273-301. http://scentsoc.org/Volumes/JAE/v15/4/00154273.pdf 
Kojima, K. I. 1971. Stochastic models for efficient control of insect populations by sterile-insect release methods, pp. 477-487. In Proceedings, Symposium: Sterility Principle for Insect Control or Eradication. International Atomic Energy Agency and Food and Agriculture Organization of the United Nations, 14-18 September 1970, Athens, Greece. STI/PUB/265. IAEA, Vienna, Austria. https://www.iaea.org/publications/3003/sterility-principles-for-insect-control-or-eradication

Lawson, F. R. 1967. Theory of control of insect populations by sexually sterile males. Annals of the Entomological Society of America 60: 713-722.

Levins, R., and D. Culver. 1971. Regional coexistence of species and competition between rare species. Proceedings of the National Academy of Science 68: 1246-1248.

Lewis, M. A., and P. van den Driessche. 1993. Waves of extinction from sterile insect release. Mathematical Biosciences 116: 221-247.

Manoranjan, V. S., and P. van den Driessche. 1986. On a diffusion model for sterile release. Mathematical Biosciences 79: 199-208.

Marsula, R., and C. Wissel. 1994. Insect pest control by a spatial barrier. Ecological Modelling 75: 203-211.

Meats, A. 1998. Predicting or interpreting trap catches resulting from natural propagules or releases of sterile fruit flies. An actuarial and dispersal model tested with data on Bactrocera tryoni. General and Applied Entomology 28: 29-38.

Meats, A., and C. J. Smallridge. 2007. Short- and long-range dispersal of medfly, Ceratitis capitata (Dipt., Tephritidae), and its invasive potential. Journal of Applied Entomology 131: 518-523. http://onlinelibrary.wiley.com/doi/10.1111/j.1439-0418.2007.01168.x/full

Miller, D. R., and D. E. Weidhaas. 1974. Equilibrium populations during a sterile-male release programme. Environmental Entomology 3: 211-216.

Mueller, L. D., and A. Joshi. 2000. Stability in model populations. Monographs in population biology 31. Princeton University Press, Princeton, NJ, USA.

Okubo, A. 1980. Diffusion and ecological problems: mathematical models. Springer, New York, USA.

Patil, G. P., and W. M. Stiteler. 1974. Concepts of aggregation and their quantification: a critical review with some new results and applications. Researches on Population Ecology 15: 238-254.

Pielou, E. C. 1969. An introduction to mathematical ecology. John Wiley, New York, USA.

Plant, R. E., and R. T. Cunningham. 1991. Analyses of the dispersal of sterile Mediterranean fruit flies (Diptera: Tephritidae) released from a point source. Environmental Entomology 20: 1493-1503.

Potgieter, L., J. H. van Vuuren, and D. E. Conlong. 2013. A reaction-diffusion model for the control of Eldana saccharina Walker in sugarcane using the sterile insect technique. Ecological Modelling 250: 319-328. https://doi.org/10.1016/j.ecolmodel.2012.11.019

Potgieter, L., J. H. Van Vuuren, and D. E. Conlong. 2016. Simulation modelling as a decision support in developing a sterile insect-inherited sterility release strategy for Eldana saccharina (Lepidoptera: Pyralidae). Florida Entomologist 99(Special Issue 1): 13-22. https://journals.flvc.org/flaent/article/view/88481

Prout, T. 1978. The joint effects of the release of sterile males and immigration of fertilized females on a density regulated population. Theoretical Population Biology 13: 40-71.

Ramirez, S., and L. F. Gordillo. 2016. Approximating optimal release in a deterministic model for the sterile insect technique. International Journal of Agronomy 2016, Article ID 8492107. 7 pp. http://dx.doi.org/10.1155/2016/8492107

Rendón, P., D. McInnis, D. Lance, and J. Stewart. 2004. Medfly (Diptera: Tephritidae) genetic sexing: large-scale field comparison of males-only and bisexual sterile fly releases in Guatemala. Journal of Economic Entomology 97: 1547-1553. https://doi.org/10.1603/0022-0493-97.5.1547

Sawyer, A. J., Z. Feng, C. W. Hoy, R. L. James, S. E. Naranjo, S. E. Webb, and C. Welty. 1987. Instructional simulation: sterile insect release method with spatial and random effects. American Entomologist 33: 182-190. https://doi.org/10.1093/besa/33.3.182

Shelly, T. E. 1997. Selection for non-responsiveness to methyl eugenol in male oriental fruit flies (Diptera: Tephritidae). Florida Entomologist 80: 248-253. http://journals.fcla.edu/flaent/article/view/74761/72419

Shiga, M. 1986. Analysis of spatial distribution in fruit fly eradication, pp. 387-398. In M. Mangel, J. R. Carey and R. E. Plant (eds.), Proceedings: Pest Control: Operations and Systems Analysis in Fruit Fly Management. NATO Advanced Workshop, 5-9 August 1985, Bad Windsheim, Germany. Springer-Verlag, Berlin, Germany. 
Staley, D. H., W. N. Cannon, Jr., and W. R. Holt. 1971. A mathematical model of an insect population with overlapping generations where the females are polyandrous and the males are subject to auto-sterilization. Annals of the Entomological Society of America 64: 325-330.

Steiner, L. F., E. J. Harris, W. C. Mitchell, M. S. Fujimoto, and L. D. Christenson. 1965. Melon fly eradication by overflooding with sterile flies. Journal of Economic Entomology 58: 519-522.

Suckling, D. M., A. M. Barrington, A. Chhagan, A. E. A. Stephens, G. M. Burnip, J. G. Charles, and S. L. Wee. 2007. Eradication of the Australian painted apple moth Teia anartoides in New Zealand: trapping, inherited sterility, and male competitiveness, pp. 603-615. In M. J. B. Vreysen, A. S. Robinson and J. Hendrichs (eds.), Area-wide control of insect pests. From research to field implementation. Springer, Dordrecht, The Netherlands. http://www-naweb.iaea.org/nafa/ipc/public/Area-Wide-Control-Insect-Pests-book.pdf

Suckling, D. M., B. Woods, V. J. Mitchell, A. Twidle, I. Lacey, E. B. Jang, and A. R. Wallace. 2011. Mobile mating disruption of light-brown apple moths using pheromone-treated sterile Mediterranean fruit flies. Pest Management Science 67: 1004-1014. http://onlinelibrary.wiley.com/doi/10.1002/ps.2150/abstract

Suckling, D. M., P. C. Tobin, D. G. McCullough, and D. A. Herms. 2012. Combining tactics to exploit Allee effects for eradication of alien insect populations. Journal of Economic Entomology 105: 1-13. https://doi.org/10.1603/EC11293

Taylor, C. R. 1976. Determining optimal sterile male release strategies. Environmental Entomology 5: 87-95.

Toledo, J., S. Flores, S. Campos, A. Villaseñor, W. Enkerlin, P. Liedo, A. Valle, and P. Montoya. 2017. Pathogenicity of three formulations of Beauveria bassiana and efficacy of autoinoculation devices and sterile fruit fly males for dissemination of conidia for the control of Ceratitis capitata. Entomologia Experimentalis et Applicata 164: 340-349. http://onlinelibrary.wiley.com/doi/10.1111/eea.12608/full

Turchin, P. 1998. Quantitative analysis of movement: measuring and modeling population redistribution in animals and plants. Sinauer Associates, New York, USA.

Villatoro, C., O. Bolanos, E. Recinos, and E. Alvarado. 2014. Evaluacion de la dispersion de mosca esteril de Ceratitis capitata (Wied.), (Diptera: Tephritidae) en la Finca La Mosqueta, Municipio de Barillas, Huehuetenango. Technical Report, Moscamed Program Guatemala. Ciudad de Guatemala.

Von Borstel, R. C., and E. F. Knipling. 1960. Population control by release of irradiated males. Science 131(3403): 878, 880-882. http://science.sciencemag.org/content/131/3403/878.1 DOI: 10.1126/science.131.3403.878 http://www.jstor.org/stable/1705645

Vreysen, M. J. B., H. J. Barclay, and J. Hendrichs. 2006. Modeling of preferential mating in areawide control programs that integrate the release of strains of sterile males only or both sexes. Annals of the Entomological Society of America 99: 607-616. https://doi.org/10.1603/0013-8746(2006)99[607:MOPMIA]2.0.CO;2

Wall, R., and J. Howard. 1994. Autosterilization for control of the housefly Musca domestica. Journal of Theoretical Biology 171: 431-437.

Wehrhahn, C. F. 1973. An approach to modelling spatially heterogeneous populations and the simulation of populations subject to sterile insect release programs, pp. 45-64. In Proceedings, Panel: Computer Models and Application of the Sterile-Male Technique. Joint FAO/IAEA Division of Atomic Energy in Food and Agriculture, 13-17 December 1971, Vienna, Austria. STI/PUB/340. IAEA, Vienna, Austria. https://www.iaea.org/publications/3096/computer-models-and-applicationof-the-sterile-male-technique-vienna-13-17-dec-1971

Weidhaas, D. E. 1973. Principles of the sterile male technique with emphasis on ecology, pp. 17-22. In Proceedings, Panel: Computer Models and Application of the Sterile-Male Technique. Joint FAO/IAEA Division of Atomic Energy in Food and Agriculture, 13-17 December 1971, Vienna, Austria. STI/PUB/340. IAEA, Vienna, Austria. https:/www.iaea.org/publications/3096/computermodels-and-application-of-the-sterile-male-technique-vienna-13-17-dec-1971

Yamanaka, T., and A. M. Liebhold. 2009. Spatially implicit approaches to understand the manipulation of mating success for insect invasion management. Population Ecology 51: 427-444. https://www.nrs.fs.fed.us/pubs/jrnl/2009/nrs_2009_yamanaka_002.pdf

Zouros, E. 1969. On the role of female monogamy in the sterile male technique of insect control. Annales de 1'Institut Phytopathologique Benaki 9: 20-29. 